\title{
Semigroup actions on tori and stationary measures on projective spaces
}

\author{
by \\ Yves Guivarc'h (Rennes) and Roman Urban (Wrocław) \\ Dedicated to Hillel Furstenberg on the occasion of \\ his 70th birthday, with admiration
}

\begin{abstract}
Let $\Gamma$ be a subsemigroup of $G=\mathrm{GL}(d, \mathbb{R}), d>1$. We assume that the action of $\Gamma$ on $\mathbb{R}^{d}$ is strongly irreducible and that $\Gamma$ contains a proximal and quasi-expanding element. We describe contraction properties of the dynamics of $\Gamma$ on $\mathbb{R}^{d}$ at infinity. This amounts to the consideration of the action of $\Gamma$ on some compact homogeneous spaces of $G$, which are extensions of the projective space $\mathbb{P}^{d-1}$. In the case where $\Gamma$ is a subsemigroup of $\operatorname{GL}(d, \mathbb{R}) \cap \mathrm{M}(d, \mathbb{Z})$ and $\Gamma$ has the above properties, we deduce that the $\Gamma$-orbits on $\mathbb{T}^{d}=\mathbb{R}^{d} / \mathbb{Z}^{d}$ are finite or dense.
\end{abstract}

1. Introduction and main results. Let $\Gamma$ be a multiplicative semigroup of integers. The semigroup $\Gamma$ is said to be lacunary if the members $\{\gamma \in \Gamma: \gamma>0\}$ are of the form $\gamma_{0}^{k}, k \in \mathbb{N}, \gamma_{0} \in \mathbb{N}^{*}$. Otherwise $\Gamma$ is non-lacunary. In 1967 Furstenberg [12] proved that if $\Gamma$ is a non-lacunary semigroup of integers and $\alpha$ is an irrational number, then the orbit $\Gamma \alpha$ is dense modulo 1 . The problem of approximating a number $\theta$ modulo 1 by numbers of the form $q \alpha$, where $\alpha$ is a fixed irrational and $q$ varies in a specified subset $Q \subset \mathbb{N}$, was considered by Hardy and Littlewood in [20] for various subsets $Q$ of $\mathbb{N}$. In particular, the result of Furstenberg above can be considered as a generalization of a theorem of [20], which asserts that if $r$ is a positive integer and $\alpha$ is an irrational number, then the set $\left\{q^{r} \alpha: q \in \mathbb{N}\right\}$ is dense modulo 1 ; furthermore, this result draws attention to the role of the multiplicative structure of $Q$ in Diophantine approximation, hence of the role of the corresponding dynamical properties of endomorphisms of $\mathbb{T}=\mathbb{R} / \mathbb{Z}$. Hence, one is led, more generally, to consider separately

2000 Mathematics Subject Classification: 54H20, 37C85, 60B11, 60J05.

Key words and phrases: asymptotic set, proximal and quasi-expanding element, toral automorphism, ID-property, random walk, projective space, stationary measure.

The second author was partially supported by RTN Harmonic Analysis and Related Problems contract HPRN-CT-2001-00273-HARP and in part by KBN grant 1P03A01826. 
the properties depending on the multiplicative structure of $\Gamma$ and the properties depending on the additive structure implied in reduction modulo 1. In this direction, a generalization of Furstenberg's result to a commutative semigroup $\Gamma \subset \mathrm{M}_{\text {inv }}(d, \mathbb{Z}):=\mathrm{GL}(d, \mathbb{R}) \cap \mathrm{M}(d, \mathbb{Z})$, where $\mathrm{M}(d, \mathbb{Z})$ is the set of $d \times d$ matrices with integer entries, acting by endomorphisms on the torus $\mathbb{T}^{d}=\mathbb{R}^{d} / \mathbb{Z}^{d}$ was given by Berend in [4].

Following [4], we say that the semigroup of endomorphisms of a $d$-dimensional torus $\mathbb{T}^{d}$ has the ID-property (cf. $[4,25,26]$ ) if the only infinite closed $\Gamma$-invariant subset of $\mathbb{T}^{d}$ is $\mathbb{T}^{d}$ itself. (ID stands for infinite invariant is dense.) Berend [4] gave necessary and sufficient conditions in arithmetical terms for a commutative semigroup to have the ID-property.

On the other hand, starting from [4] and [12], Margulis [24] asked for necessary and sufficient conditions on a subsemigroup $\Gamma \subset \mathrm{M}_{\text {inv }}(d, \mathbb{Z})$ in order that the $\Gamma$-orbit closures on $\mathbb{T}^{d}$ are finite unions of manifolds. We observe that it follows from general results of Dani and Raghavan on linear actions [9] that the orbits of $\Gamma=\operatorname{SL}(d, \mathbb{Z})$ acting on $\mathbb{T}^{d}$ are finite or dense. In this direction a detailed study of $\Gamma$-orbits in $\mathbb{R}^{d}$ of a general subgroup $\Gamma \subset$ $\mathrm{SL}(d, \mathbb{R})$ was developed by Conze and Guivarc'h in [7]. The homogeneity at infinity of $\Gamma$-orbits was pointed out there as well as the role of " $\Gamma$-irrational" vectors in the construction of limit points of $\Gamma$-orbits, if $\Gamma$ is a general subgroup of $\mathrm{SL}(d, \mathbb{R})$.

Some results in the direction of the general question of Margulis have been obtained recently. Muchnik proved in [25] that if the semigroup $\Gamma$ of $\operatorname{SL}(d, \mathbb{Z})$ is Zariski dense in $\operatorname{SL}(d, \mathbb{R})$, then $\Gamma$ acting on $\mathbb{T}^{d}$ has the ID-property. In [29] Starkov proved the same result in case $\Gamma$ is a strongly irreducible subgroup of $\mathrm{SL}(d, \mathbb{Z})$. In the next paper [26] Muchnik generalized the results of Berend to semigroups of $\mathrm{M}_{\mathrm{inv}}(d, \mathbb{Z})$. At the same time Guivarc'h and Starkov [19] derived an important part of Muchnik's result using different methods, based on $[6,7]$. We observe that in [19], the property $\Gamma \subset \mathrm{SL}(d, \mathbb{Z})$ is used only when additive aspects connected with reduction modulo one come into play. It turned out that the property of $\Gamma$-orbits in $\mathbb{R}^{d}$ which is responsible for density in $\mathbb{T}^{d}$ is "thickness" at infinity of $\Gamma$-orbits (see Theorem 5.23 and the comments to it). Hence, this property can be studied separately in full generality; $\Gamma$ is then a general subsemigroup of $\mathrm{GL}(d, \mathbb{R})$ and the use of boundaries and random walks is natural in this context.

In this paper we consider this problem in a simplified setting, we give a self-contained exposition of some of the methods developed in $[6,7,19]$ in the more general context of random walks and linear actions, and we use the results to prove the ID-property in our setting. We also prove some new results for actions on tori and on certain compact $G$-factor spaces of $\mathbb{R}^{d}$.

The general idea is to lift the automorphisms of the torus $\mathbb{T}^{d}$ to its universal cover $\mathbb{R}^{d}$ and to study the action of the lifts at infinity. The action 
of $\Gamma$ at infinity can be expressed in terms of some compact homogeneous spaces of $\mathrm{GL}(d, \mathbb{R})$ which are closely related to the projective spaces $\mathbb{P}^{d-1}$. The random walk framework allows us to take into account the global semigroup asymptotic behavior in terms of stationary measures and convergence to them. As in [13] and [15], the results can be used to obtain topological properties of the $\Gamma$-action. Furthermore, this general framework allows us to obtain a series of facts about linear actions which are of interest in other problems.

Before we state the results we need to introduce some notions. A matrix $\gamma \in \operatorname{GL}(d, \mathbb{R})$ is said to be proximal if it has an eigenvalue $\lambda_{\gamma}$ such that $\left|\lambda_{\gamma}\right|>|\lambda|$ for all other eigenvalues $\lambda$ of $\gamma$. A matrix $\gamma$ is said to be quasiexpanding if it has an eigenvalue $\lambda$ such that $|\lambda|>1$.

Let $\Gamma$ be a subsemigroup of $\mathrm{GL}\left(d, \mathbb{R}\right.$ ). The $\Gamma$-action on $\mathbb{R}^{d}$ (or simply $\Gamma$ ) is said to be strongly irreducible if no finite union of proper subspaces is $\Gamma$-invariant.

The first main theorem of this paper is as follows:

THEOREM 1.1. Let $\Gamma$ be a subsemigroup of $\mathrm{M}_{\mathrm{inv}}(d, \mathbb{Z}), d>1$, such that $\Gamma$ contains a proximal element and the $\Gamma$-action on $\mathbb{R}^{d}$ is strongly irreducible. Then the semigroup $\Gamma$ acting on $\mathbb{T}^{d}$ has the ID-property, that is, every infinite $\Gamma$-invariant subset of $\mathbb{T}^{d}$ is dense.

If $d=1$, one has $\mathrm{M}_{\text {inv }}(1, \mathbb{Z})=\mathbb{Z}^{*} \subset \mathbb{R}^{*}$. As said above, the conclusion of Theorem 1.1 is valid in this case too, if and only if $\Gamma$ is non-lacunary, i.e., not contained in a cyclic subgroup of $\mathbb{R}^{*}$. For $d>1$, the condition in Theorem 1.1 implies that $\Gamma$ is not contained in a finite extension of an abelian subgroup of $\mathrm{M}_{\text {inv }}(d, \mathbb{Z})$; in particular, here $\Gamma$ is non-abelian, hence the situation of [4] is excluded from our setting.

The first step in order to get Theorem 1.1 is to study closed infinite $\Gamma$-invariant subsets $\Sigma$ of $\mathbb{T}^{d}$ such that 0 is a limit point of $\Sigma$. Then we notice that the inverse image in $\mathbb{R}^{d}$ of such an infinite $\Gamma$-invariant subset contains some asymptotic set which consists of lines. Moreover, there are some rays with good properties, that is, not contained in a subspace having a basis which consists of integer vectors. This allows us to project them using the canonical projection $p: \mathbb{R}^{d} \rightarrow \mathbb{R}^{d} / \mathbb{Z}^{d}, p(x)=x+\mathbb{Z}^{d}$, on $\mathbb{T}^{d}$ and obtain the result in the case when 0 is a limit point of the subset $\Sigma$. Furthermore, using arguments close to [4] and [12] and reduction to a finitely generated subsemigroup of $\Gamma$, we show that the opposite situation does not occur.

Let $L_{\Gamma} \subset \mathbb{P}^{d-1}$ be the closure of the set of directions corresponding to dominant eigenvectors of the proximal elements in $\Gamma$. We denote by $\widetilde{L}_{\Gamma}$ the set of corresponding non-zero vectors in $V=\mathbb{R}^{d}$, by $\sigma$ the symmetry $\sigma: v \mapsto-v$ in $V$, and by $\widetilde{V}$ the factor space $\widetilde{V}=V /\{\sigma, \operatorname{Id}\}$.

The following is the basic tool in the proof of Theorem 1.1. 
TheOREM 1.2. Suppose $\Gamma$ is a subsemigroup of $\mathrm{GL}(d, \mathbb{R}), d>1$, which is strongly irreducible and contains a proximal and quasi-expanding element. Let $\Sigma$ be a $\Gamma$-invariant subset of $\widetilde{V} \backslash\{0\}$ and suppose $0 \in \bar{\Sigma}$. Then

$$
\bar{\Sigma} \supset \widetilde{L}_{\Gamma} /\{\sigma, \operatorname{Id}\} \text {. }
$$

To have in mind a simple example illustrating Theorems 1.1 and 1.2, consider the torus $\mathbb{T}^{2}$. One of the simplest examples of a subsemigroup of $\operatorname{SL}(2, \mathbb{Z})$ satisfying the conditions of Theorem 1.1 is the semigroup $\Gamma=\langle a, b\rangle$ generated by the matrices $a=\left(\begin{array}{ll}2 & 1 \\ 1 & 1\end{array}\right)$ and $b=\left(\begin{array}{ll}3 & 2 \\ 1 & 1\end{array}\right)$ from $\mathrm{SL}(2, \mathbb{Z})$.

From Theorem 1.1 we infer that the $\Gamma$-orbits in $\mathbb{T}^{2}$ are finite or dense. Furthermore we observe that, in the context of Theorem 1.2, the dynamics of $\Gamma$ on $\mathbb{R}^{2}$ is easy to visualize. The closure of the eigen-directions in the positive quadrant $\mathbb{R}_{+}^{2}$ forms a Cantor set and the corresponding lines form an "attractor set" $\widetilde{L}_{\Gamma}^{1}$ for the action of $\Gamma$ in $\mathbb{R}_{+}^{2}$. There exist vectors in $\mathbb{R}^{2}$ whose orbit closures contain 0 , for example dominant eigenvectors of elements of $\Gamma^{-1}$. The $\Gamma$-orbit for such a vector tends to fill $\widetilde{L}_{\Gamma}^{1} \cup-\widetilde{L}_{\Gamma}^{1}$ since the dynamics of its $\Gamma$-orbit consists of attraction towards 0 and expansion along the eigenvectors sitting in $\widetilde{L}_{\Gamma}^{1} \cup-\widetilde{L}_{\Gamma}^{1}$.

For a general vector, for example a vector $v \in \mathbb{R}_{+}^{2}$, there is attraction towards $\widetilde{L}_{\Gamma}^{1}$ and expansion along $\widetilde{L}_{\Gamma}^{1}$, and the $\Gamma$-orbit of $v$ is "thick at infinity" due to the irrationality properties of eigenvalues of elements in $\Gamma$. In the general case the situation is similar, in particular the projections of general $\Gamma$-orbits into $\mathbb{T}^{d}$ are large, hence one can expect the closed $\Gamma$-orbits in $\mathbb{T}^{d}$ to be finite unions of special manifolds, as conjectured in [24].

Let us now consider, for $c>1$, the factor space $\mathbb{P}_{c}^{d-1}$ of $V \backslash\{0\}$ by the subgroup of homotheties with ratio $\pm c^{k}(k \in \mathbb{Z})$. The action of $g \in G=$ $\operatorname{GL}(d, \mathbb{R})$ on $v \in \mathbb{P}_{c}^{d-1}$ will be denoted $v \mapsto g . v$. Let $\mu$ be a probability measure on $\Gamma \subset \mathrm{GL}(d, \mathbb{R})$ whose support generates $\Gamma$. Then we can define an associated Markov operator $P_{\mu}$ on $\mathbb{P}_{c}^{d-1}$ by the formula

$$
P_{\mu}(v, \cdot)=\int \delta_{g \cdot v} d \mu(g) .
$$

The iterates $P_{\mu}^{n}$ of $P_{\mu}$ define a random walk on $\mathbb{P}_{c}^{d-1}$.

The following describes the asymptotic behavior of the iterates $P_{\mu}^{n}$; it is an essential tool in the proof of Theorem 1.2, hence of Theorem 1.1.

TheOREM 1.3. Assume that $\Gamma \subset \mathrm{GL}(d, \mathbb{R})$ is a subsemigroup which is strongly irreducible and contains a proximal element. With the above notations, the Markov operator $P_{\mu}$ on $\mathbb{P}_{c}^{d-1}$ has a unique stationary measure $\varrho$, the support $S_{\varrho}$ of $\varrho$ is the unique closed $\Gamma$-invariant minimal subset of $\mathbb{P}_{c}^{d-1}$, and for any $v \in \mathbb{P}_{c}^{d-1}$ the sequence of measures $P_{\mu}^{n}(v, \cdot)$ converges to $\varrho$. Moreover, the trajectories of $P_{\mu}$ starting from $v$ converge a.e. to $S_{\varrho}$. 
Along the way, we get some new results and facts. For example, we show a priori that the weak ID-property (that is, the closures of the orbits $\Gamma x$, $x \in \mathbb{T}^{d}$, are either finite or equal to $\mathbb{T}^{d}$ itself) and the ID-property are equivalent, a fact implicitly used in previous papers, but apparently unproved in the literature.

We also clarify the relations between a fundamental cocycle equation on $\Gamma \times \mathbb{P}^{d-1}$ and an aperiodicity condition for the dominant eigenvalues of proximal elements in $\Gamma$ which occurs in [22] and which also has a geometric interpretation in terms of lengths of closed geodesics (see [8]).

Furthermore, the result in Theorem 1.3 extends results of [17] but is new in this generality.

Also the result of Theorem 1.1 is not covered by [19] since, in our setting, $\Gamma$ is allowed to be a subsemigroup of $\mathrm{M}_{\text {inv }}(d, \mathbb{Z})(d>1)$. We are led to prove a result of independent interest: $\Gamma$ can be supposed to be finitely generated (see Proposition 2.6).

The structure of the paper is as follows. In Section 2 we set the notation and give all necessary definitions. In particular, we define a dominant vector, a proximal element and state our two hypothesis $\left(H_{1}\right)$ (strong irreducibility) and $\left(H_{2}\right)$ (proximality), under which we prove Theorem 1.3. We introduce hypothesis $\left(H_{0}\right)$, i.e. the unboundedness of $\Gamma$-orbits in $V \backslash\{0\}$. Under $\left(H_{1}\right)$ and $\left(H_{2}\right)$, this condition is equivalent (see Proposition 2.4) to the existence of a proximal and quasi-expanding element in $\Gamma$, which allows us to prove Theorems 1.1 and 1.2. It is clear that this condition is necessary for the validity of the ID-property.

We observe that conditions $\left(H_{0}\right),\left(H_{1}\right)$ and $\left(H_{2}\right)$ are analogous to those used in [16, Theorem 2.5] in order to get a homogeneous behavior at infinity of the potential measure in $\widetilde{V}$ associated with $\mu$, hence also of the $\Gamma$-orbits at infinity in $\widetilde{V}$. (See also [10] for the case of affine actions.)

In Section 3 we prove the equivalence of the weak ID-property and IDproperty (Proposition 3.1).

In Section 4 we study the $\Gamma$-actions on various spaces, namely on the projective space $\mathbb{P}(V)$, the compact homogeneous space $\mathbb{P}_{c}(V)$ and $V$ itself. We define the asymptotic sets for $\Gamma$-actions and we study their properties. We also clarify the role of aperiodicity hypotheses of $\Gamma$ considered by Kesten in [22] and Eberlein in [11] (see Corollary 4.8 and Proposition 4.6).

Section 5 develops the random walks techniques which are used in the proof of the main new result of this section which is Theorem 5.19. This theorem together with the method presented in [12] allow us to prove Theorem 1.1 in Section 6. Theorem 5.19 follows from a detailed study of random walks on $V$ and various $\Gamma$-spaces, governed by a measure $\mu$ sitting on $\Gamma$ and such that the convolution iterations $\mu^{* k}$ fill $\Gamma$. Some of these results 
are well known but we have included the proofs in order to make the paper self-contained. Some others are new.

Finally, in Section 6 we give the proof of Theorem 1.1.

2. Proximality, irreducibility, expansivity. In what follows, $\Gamma$ will denote a subsemigroup of $\mathrm{GL}(d, \mathbb{R})$. We consider the actions of $\Gamma$ on the vector space $V=\mathbb{R}^{d}$, on the associated projective space $\mathbb{P}^{d-1}=\mathbb{P}(V)$, and on $\widetilde{V}=V /\{\operatorname{Id}, \sigma\}=V /\{ \pm \mathrm{Id}\}$. We denote by $\pi$ the projection of $V \backslash\{0\}$ on $\mathbb{P}^{d-1}=\mathbb{P}(V)$ and we identify $\mathbb{P}(V)$ with the unit sphere $\mathbb{S}^{d-1}$ divided by the symmetry $\sigma: x \mapsto-x$. Also $K=\mathrm{SO}(d, \mathbb{R})$ will denote the special orthogonal group and $m$ the unique $K$-invariant probability measure on $\mathbb{P}(V)$.

The action of the matrix $g$ on the vector $x \in V$ is denoted by $(g, x) \mapsto g x$, whereas for the action of $g$ on the projective space $\mathbb{P}(V)$ we write $g . \pi(x)=$ $\pi(g x)$.

A matrix $\gamma \in \mathrm{GL}(d, \mathbb{R})$ is said to be proximal if it has an eigenvalue $\lambda_{\gamma}$ such that $\left|\lambda_{\gamma}\right|>|\lambda|$ for all other eigenvalues $\lambda$ of $\gamma$. Thus $\lambda_{\gamma} \in \mathbb{R}$. For such a $\gamma$ an eigenvector $v_{\gamma} \in V$ corresponding to the eigenvalue $\lambda_{\gamma}$ is called a dominant eigenvector or simply dominant vector of $\gamma$. By $\Delta_{\Gamma}$ we denote the set of all proximal elements in $\Gamma$. An element $\gamma \in \mathrm{GL}(d, \mathbb{R})$ is said to be quasi-expanding if it has an eigenvalue $\lambda$ such that $|\lambda|>1$.

More generally, for $u \in \operatorname{End}(V)$, we denote by $\left|\lambda_{u}\right|$ the spectral radius of $u$.

If $\gamma \in \Delta_{\Gamma}$ then we define $\gamma^{+} \in \mathbb{P}(V)$ as a point corresponding to the line in $V$ generated by $v_{\gamma}$. By $V_{\gamma}^{<}$we denote the unique $\gamma$-invariant hyperplane complementary to $V_{\gamma}^{\max }=\mathbb{R} v_{\gamma}$.

We consider the following assumptions.

$\left(H_{0}\right) \quad$ For every $v \in V \backslash\{0\}$, the orbit $\Gamma v$ is unbounded.

$\left(H_{1}\right) \quad$ The $\Gamma$-action is strongly irreducible (for short, $\Gamma$ is strongly irreducible), that is, no finite union of proper subspaces is $\Gamma$-invariant.

$\left(H_{2}\right) \quad \Gamma$ contains a matrix $\gamma$ which is proximal.

REMARK 2.1. (i) Condition $\left(H_{1}\right)$ can be equivalently formulated as follows. A subsemigroup $\Gamma$ of $\mathrm{GL}(V)$ acts strongly irreducibly on $V$ if every finite index subgroup $H$ of the group $\left\langle\Gamma, \Gamma^{-1}\right\rangle$ acts irreducibly on $V$, that is, every $H$-invariant subspace of $V$ is either 0 or $V$.

(ii) If $\Gamma$ is a subsemigroup of $\mathrm{SL}(d, \mathbb{R})$, then conditions $\left(H_{1}\right)$ and $\left(H_{2}\right)$ imply $\left(H_{0}\right)$, since otherwise the determinant of the proximal element $\gamma$ would be strictly less than 1 . The same is true, using the same argument, if $\Gamma$ is a subsemigroup of $\mathrm{M}_{\text {inv }}(d, \mathbb{Z})$, since $\operatorname{det} \gamma, \gamma \in \Gamma$, is a nonzero integer (see Proposition 2.4 below).

(iii) Condition $\left(H_{1}\right)$ (resp. $\left.\left(H_{2}\right)\right)$, if valid for $\Gamma$, is also valid for $\Gamma^{t}$, the transposed semigroup acting on the dual space $V^{*}$. 
(iv) Conditions $\left(H_{0}\right),\left(H_{1}\right)$ for $\Gamma$ imply condition $\left(H_{0}\right)$ for $\Gamma^{t}$. This will be used in the proof of Theorem 1.1 and can be seen as follows. Let $W \subset V^{*}$ be the subspace of vectors with bounded $\Gamma^{t}$-orbits. Then $W$ is $\Gamma^{t}$-invariant, hence (iii) implies $W=\{0\}$ or $W=V^{*}$. In case $W=V^{*}, \Gamma^{t}$ is relatively compact in $\operatorname{End}\left(V^{*}\right)$, hence $\Gamma$ is relatively compact in $\operatorname{End}(V)$. This contradicts condition $\left(H_{0}\right)$ for $\Gamma$.

The concept of Zariski closure, defined below, will be freely used when dealing with the above conditions (see [27]).

Let $\Gamma$ be a subset of $\mathrm{GL}(d, \mathbb{R})$. We recall that the Zariski closure $\mathrm{Zc}(\Gamma)$ of $\Gamma$ is the set of zeros of all real polynomials in the coefficients of $g \in \operatorname{GL}(d, \mathbb{R})$ and $(\operatorname{det} g)^{-1}$, which vanish on $\Gamma$.

If $\Gamma$ is a subsemigroup of $\operatorname{GL}(d, \mathbb{R})$ then $\mathrm{Zc}(\Gamma)$ is a group which contains $\Gamma$, is closed and has a finite number of connected components in the real topology (see [27]). The connected component of the identity in the Zariski topology is a subgroup of finite index which will be denoted by $\mathrm{Zc}_{0}(\Gamma)$.

We have the following generalization of Lemma 2.8 in [6] to the case of semigroups.

Lemma 2.2. Let $\Gamma \subset \mathrm{GL}(V)$ be a subsemigroup. The $\Gamma$-action satisfies condition $\left(H_{1}\right)$ if and only if the orbit $\Gamma v$ of no non-zero vector $v$ is contained in a finite union of proper vector subspaces of $V$.

Proof. Suppose $\left(H_{1}\right)$ to be valid and $v \in V$ be such that $\Gamma v \subset \bigcup_{j=1}^{n} V_{j}$ where $V_{j}$ are proper subspaces of $V$. Let $W$ be a finite union of subspaces of $V$ such that $\Gamma v \subset W$, and $\mathcal{W}$ the set of such $W$. We observe that $\Gamma v \subset$ $\bigcap_{W \in \mathcal{W}} W$. Since a strictly decreasing family of elements of $\mathcal{W}$ is finite we see that $\bigcap_{W \in \mathcal{W}} W$ also belongs to $\mathcal{W}$, in other words $W_{0}:=\bigcap_{W \in \mathcal{W}} W$ is the minimum element in $\mathcal{W}$. We write $W_{0}=\bigcup_{j=1}^{m} V_{j}$; we are going to show that $W_{0}$ is preserved by $\Gamma$. Since $W \in \mathcal{W}$ is algebraic we have $\operatorname{Zc}(\Gamma) v \subset W$, in particular $\left\langle\Gamma, \Gamma^{-1}\right\rangle v \subset W$. It follows that, for any $\gamma \in \Gamma$,

$$
\gamma W \supset \gamma\left\langle\Gamma, \Gamma^{-1}\right\rangle v \supset \Gamma v .
$$

Hence, $\gamma W \in \mathcal{W}$. Since $W_{0}$ is the minimum element of $\mathcal{W}$, we have $\gamma W_{0} \supset$ $W_{0}$, so $\gamma W_{0}=W_{0}$; hence, $\Gamma W_{0}=W_{0}$. Condition $\left(H_{1}\right)$ says that this is impossible.

Conversely, suppose $V_{j}(1 \leq j \leq n)$ is a family of proper subspaces which is preserved by $\Gamma$. Let $v \in V_{1}$; then $\Gamma v \in \bigcup_{i=1}^{n} V_{i}$. From the hypothesis this is impossible, hence condition $\left(H_{1}\right)$ is satisfied.

Let $X$ be a compact metric space with distance function $\delta$. We say that the action of a semigroup $\Gamma$ of continuous transformations of $X$ is proximal if, given $x, y \in X$, there exists a sequence $\left\{\gamma_{n}\right\} \subset \Gamma$ such that $\delta\left(\gamma_{n} . x, \gamma_{n} . y\right) \rightarrow 0$ as $n \rightarrow \infty$. 
Define the distance function $\delta$ on $\mathbb{P}(V)$ as follows:

$$
\delta(\bar{u}, \bar{v})=\|u \wedge v\| /\|u\|\|v\|, \quad \bar{u}, \bar{v} \in \mathbb{P}(V),
$$

where $u$ and $v$ are the corresponding vectors in the vector space $V$.

Proposition 2.3 (Theorem 2.9 in [14]). Let $\Gamma$ be a subsemigroup of $\mathrm{GL}(V)$. Then the following are equivalent:

(a) $\Gamma$ satisfies $\left(H_{1}\right)$ and $\left(H_{2}\right)$.

(b) $\Gamma$ acts proximally on $\mathbb{P}(V)$ and is strongly irreducible.

Proof. $((\mathrm{a}) \Rightarrow(\mathrm{b}))$ We consider a proximal element $\gamma \in \Gamma$ and define

$$
u=\lim _{n}\left\|\gamma^{2 n}\right\|^{-1} \gamma^{2 n}, \quad \mathfrak{z}=\operatorname{Ker} u \subset \mathbb{P}\left(V^{*}\right) .
$$

Then if $x, y \in \mathbb{P}(V)$ do not belong to $\operatorname{Ker} u$, we have $\lim _{n} \gamma^{n} \cdot x=\gamma^{+}$, $\lim _{n} \gamma^{n} \cdot y=\gamma^{+}$. Hence, $\lim _{n} \delta\left(\gamma^{n} \cdot x, \gamma^{n} \cdot y\right)=0$.

In general, if $x, y \in \mathbb{P}(V)$ are given we can find $h \in \Gamma$ such that $h . x \notin$ Ker $u$ and $h . y \notin \operatorname{Ker} u$, otherwise, passing to the dual space $V^{*}$, transposing maps, and using the hyperplanes $x^{\perp}$ and $y^{\perp}$ of $V^{*}$ defined by $x$ and $y$, one would have

$$
\forall h \in \Gamma, \quad h^{t} \cdot \mathfrak{z} \subset x^{\perp} \text { or } h^{t} \cdot \mathfrak{z} \subset y^{\perp} .
$$

But Remark 2.1(iii) and Lemma 2.2 say that this is impossible under condition $\left(H_{1}\right)$.

$((\mathrm{b}) \Rightarrow(\mathrm{a}))$ It follows from proximality of $\Gamma$ on the compact metric space $\mathbb{P}(V)$ (see [13]) that, given a finite subset $E \subset \mathbb{P}(V)$, there exist a sequence $\left\{g_{n}\right\} \subset \Gamma$ and $x \in \mathbb{P}(V)$ such that

$$
\forall y \in E, \quad \lim _{n} g_{n} \cdot y=x .
$$

We consider a finite system $E=\left\{x_{1}, \ldots, x_{2 d-1}\right\}$ of $2 d-1$ points in $\mathbb{P}(V)$ such that any $d$-subsystem consists of independent points.

We consider the linear maps $u_{n}=g_{n} /\left\|g_{n}\right\|$ and using a convergent subsequence, we can assume that $u_{n}$ converges towards $u \in \operatorname{End}(V)$ with $\|u\|=1$. We show that $u$ has rank one.

From the definition of $E$ it follows that at least $d$ points of $E$ do not belong to Ker $u$. We replace these points, as well as $x$, by the corresponding unit vectors in $V$, say $\widetilde{x}_{1}, \ldots, \widetilde{x}_{d}, \widetilde{x}$. Then we obtain

$$
u \widetilde{x}_{i}=\lambda_{i} \widetilde{x}, \quad 1 \leq i \leq d,
$$

where $\lambda_{i} \neq 0$; the points $\left\{\widetilde{x}_{i}\right\}$ form a basis of $V$, hence the rank of $u$ is one, i.e., $\operatorname{dim} \operatorname{Ker} u=d-1$. We can moreover suppose that $\operatorname{Im} u \not \subset \operatorname{Ker} u$, since otherwise we could replace $g_{n}$ by $g g_{n}$, where $g \in \Gamma$ satisfies $\operatorname{Im} g u=$ $g(\operatorname{Im} u) \not \subset \operatorname{Ker} u$ and $\operatorname{Ker} g u=\operatorname{Ker} u$. The existence of $g \in \Gamma$ such that $g(\operatorname{Im} u) \not \subset \operatorname{Ker} u=\operatorname{Ker} g u$ follows from Lemma 2.2. 
Under this condition, $u$ is proportional to the projection on $\operatorname{Im} u$ along the hyperplane Ker $u$. In particular, $u$ has a unique non-zero eigenvalue $\lambda$. Since the sequence $g_{n} /\left\|g_{n}\right\|-u$ converges to zero, we conclude that for $n$ large, $g_{n} /\left\|g_{n}\right\|$ also has a unique dominant eigenvalue close to $\lambda$. The same is true for $g_{n}$, hence $\Gamma$ satisfies $\left(H_{2}\right)$.

Proposition 2.4. Let $\Gamma$ be a subsemigroup of $\mathrm{GL}(V)$. Then the following are equivalent:

(a) $\Gamma$ satisfies $\left(H_{1}\right),\left(H_{2}\right)$ and the element $\gamma$ in condition $\left(H_{2}\right)$ satisfies $\left|\lambda_{\gamma}\right|>1$.

(b) $\Gamma$ is unbounded and satisfies $\left(H_{1}\right)$ and $\left(H_{2}\right)$.

(c) $\Gamma$ satisfies $\left(H_{0}\right),\left(H_{1}\right)$ and $\left(H_{2}\right)$.

(d) $\Gamma$ satisfies $\left(H_{1}\right),\left(H_{2}\right)$ and there exists $\gamma \in \Gamma$ such that $\left|\lambda_{\gamma}\right|>1$.

Proof. $((\mathrm{d}) \Rightarrow(\mathrm{b}))$ Let $\gamma \in \Gamma$ be a quasi-expanding element in $\Gamma$, hence $\left|\lambda_{\gamma}\right|>1$. Then $\left\|\gamma^{n}\right\| \geq\left|\lambda_{\gamma}\right|^{n}$. Hence $\lim _{n}\left\|\gamma^{n}\right\|=\infty$, i.e., $\Gamma$ is unbounded.

$((\mathrm{b}) \Rightarrow(\mathrm{a}))$ We will use the basic [1, Theorem 4.1], which allows us to construct new proximal maps and which implies the following. If $\Gamma \subset \mathrm{GL}(V)$ satisfies $\left(H_{1}\right)$ and $\left(H_{2}\right)$ there exist $\varepsilon>0, r>1$ and a finite subset $M \subset \Gamma$, such that, for any $g \in \mathrm{GL}(V)$, there exist $a \in M$ such that $a g$ is proximal, the distance in $\mathbb{P}(V)$ of $(a g)^{+}$to $V_{a g}^{<}$is at least $\varepsilon$, and

$$
\left|\lambda_{a g}\right| \geq r\left\|\left.(a g)\right|_{V_{a g}^{<}}\right\| \text {. }
$$

Since $\Gamma$ is unbounded, there exists a sequence $\left\{\gamma_{n}\right\} \subset \Gamma$ such that

$$
\lim _{n}\left\|\gamma_{n}\right\|=\infty \text {. }
$$

Using a subsequence of $\gamma_{n}$ we can suppose that, for some $a \in M, a \gamma_{n}$ is proximal, $\left(a \gamma_{n}\right)^{+},\left(V_{a \gamma_{n}}^{<}\right.$resp. $)$converges to $x \in \mathbb{P}(V)\left(W_{n}=V_{a \gamma_{n}}^{<}\right.$converges to the hyperplane $W$ of $\mathbb{P}(V)$, resp.). We have $x \notin W$, since the distance of $\left(a \gamma_{n}\right)^{+}$to $W_{n}$ is at least $\varepsilon$. We can also suppose that $a \gamma_{n} /\left\|a \gamma_{n}\right\|$ converges to $u \in \operatorname{End}(V)$ with $\|u\|=1$. Clearly, $V$ is the direct sum of the hyperplane $W$ and of the line generated by $x$. Furthermore,

$$
\left|\lambda_{u}\right|=\lim _{n} \frac{\left|\lambda_{a \gamma_{n}}\right|}{\left\|a \gamma_{n}\right\|}, \quad\left\|\left.u\right|_{W}\right\|=\lim _{n} \frac{1}{\left\|a \gamma_{n}\right\|}\left\|\left.\left(a \gamma_{n}\right)\right|_{W_{n}}\right\| .
$$

Since $u \neq 0$ preserves the above direct sum we have $\left|\lambda_{u}\right|>0$. Then the condition $\left|\lambda_{a \gamma_{n}}\right| \geq r\left\|\left.\left(a \gamma_{n}\right)\right|_{W_{n}}\right\|$ implies

$$
\left|\lambda_{u}\right| \geq r\left\|\left.u\right|_{W}\right\|, \quad\left|\lambda_{u}\right|>\left\|\left.u\right|_{W}\right\| .
$$

In particular, $u$ has a dominant eigenvalue which is simple. Since $\left\|u-\frac{a \gamma_{n}}{\left\|a \gamma_{n}\right\|}\right\|$ converges to zero, for $n$ large we have

$$
\left|\lambda_{a \gamma_{n}}\right| \geq\left\|a \gamma_{n}\right\|\left|\lambda_{u}\right| / 2
$$


Moreover, the condition $\lim _{n}\left\|\gamma_{n}\right\|=\infty$ implies

$$
\lim _{n}\left\|a \gamma_{n}\right\| \geq \lim _{n}\left\|a^{-1}\right\|^{-1}\left\|\gamma_{n}\right\|=\infty .
$$

In particular, for $n$ large, $\left|\lambda_{a \gamma_{n}}\right|>1$, hence $a \gamma_{n}$ is proximal and quasiexpanding, i.e., (a) is valid.

$((\mathrm{b}) \Rightarrow(\mathrm{c}))$ We consider the subspace $W \subset V$ of vectors in $V$ having a bounded $\Gamma$-orbit. Clearly, this subspace is $\Gamma$-invariant. Then condition $\left(H_{1}\right)$ implies $W=V$ or $W=\{0\}$. In the second case $\left(H_{0}\right)$ has been proved. The first case does not occur since it contradicts the hypothesis that $\Gamma$ is unbounded.

$((\mathrm{c}) \Rightarrow(\mathrm{b}))$ and $((\mathrm{a}) \Rightarrow(\mathrm{d}))$ are trivial.

The following is a useful characterization of strong irreducibility in terms of Zariski closure.

Proposition 2.5. Let $\Gamma$ be a subsemigroup of $\mathrm{GL}(V)$. Then $\Gamma$ satisfies $\left(H_{1}\right)$ if and only if $\mathrm{Zc}_{0}(\Gamma)$ acts irreducibly on $V$.

Proof. Assume that $\Gamma$ satisfies $\left(H_{1}\right)$ and let $W \subset V$ be a non-zero $\mathrm{Zc}_{0}(\Gamma)$-invariant subspace. For some finite set $F \subset \Gamma$ we have $\Gamma \subset \operatorname{Zc}(\Gamma)=$ $\bigcup_{\gamma \in F} \gamma \mathrm{Zc}_{0}(\Gamma)$, hence $\Gamma W=\bigcup_{\gamma \in F} \gamma W$. Since $\Gamma$ satisfies $\left(H_{1}\right)$ we get $W=V$, hence $\mathrm{Zc}_{0}(\Gamma)$ acts irreducibly on $V$.

Assume that $\mathrm{Zc}_{0}(\Gamma)$ acts irreducibly on $V$ and let $W$ be a non-zero subspace of $V$, and $F$ a finite subset of $\Gamma$ such that $\Gamma W=\bigcup_{\gamma \in F} \gamma W$. Since $\Gamma W$ is an algebraic manifold, $\mathrm{Zc}(\Gamma)$ leaves $\Gamma W$ invariant, hence permutes the subspaces $\gamma W(\gamma \in \Gamma)$. Since $\mathrm{Zc}_{0}(\Gamma)$ is connected, for any $\gamma \in F$ we have

$$
\mathrm{Zc}_{0}(\Gamma) \gamma W=\gamma W .
$$

From the irreducibility of the action of $\mathrm{Zc}_{0}(\Gamma)$ on $V$, we get $W=V$.

The following will be essential in the proof of Theorem 1.1.

Proposition 2.6. Assume that the semigroup $\Gamma \subset \mathrm{GL}(V)$ satisfies $\left(H_{1}\right)$ and $\left(H_{2}\right)$. Then $\Gamma$ contains a finitely generated subsemigroup which satisfies $\left(H_{1}\right)$ and $\left(H_{2}\right)$.

The proof of the above proposition depends on the following

Lemma 2.7. Assume that $\Gamma$ satisfies $\left(H_{1}\right),\left(H_{2}\right)$. Denote by $D$ (resp. $C$ ) the commutator subgroup (resp. connected center) of $\mathrm{Zc}_{0}(\Gamma)$. Then $\mathrm{Zc}_{0}(\Gamma)$ is the almost direct product of $D$ and $C$. Furthermore, $D$ is semisimple without compact factors and $C$ consists of homotheties.

Proof. Since $\Gamma$ acts irreducibly on $V, \operatorname{Zc}(\Gamma)$ is an $\mathbb{R}$-reductive group (see [27]), hence $D$ is semisimple and $\mathrm{Zc}_{0}(\Gamma)$ is the almost direct product of $C$ and $D$. We can write $D$ as the almost direct product $D=D_{1} D_{2}$, where 
$D_{1}$ is compact and $D_{2}$ is semisimple without compact factor. Since $\Gamma$ contains a proximal element and $\mathrm{Zc}(\Gamma) / \mathrm{Zc}_{0}(\Gamma)$ is finite, $\mathrm{Zc}_{0}(\Gamma)$ also contains a proximal element. We denote this element by $\gamma$ and write $\gamma v_{\gamma}=\lambda_{\gamma} v_{\gamma}$, $\gamma=c d_{1} d_{2}$ with $c \in C, d_{1} \in D_{1}, d_{2} \in D_{2}$. Since $d_{1}$ and $\gamma$ commute, and the direction of $v_{\gamma}$ is uniquely determined by $\gamma, d_{1} v_{\gamma}$ is proportional to $v_{\gamma}$. Since $D_{1}$ is compact we have $d_{1} v_{\gamma}= \pm v_{\gamma}$, hence $c d_{2}$ is also proximal with dominant eigenvector $v_{\gamma}$. Since $C$ commutes with $c d_{2}$, and $v_{\gamma}$ is $c d_{2}$-dominant, there exists an $\mathbb{R}$-character $\chi$ of $C$ such that $g v_{\gamma}=\chi(g) v_{\gamma}$ for every $g \in C$. Since the subspace $W=\{v: c v=\chi(c) v, \forall c \in C\}$ is $\Gamma$-invariant, contains $v_{\gamma}$, and the action of $\Gamma$ is irreducible, it follows that $c v=\chi(c) v$ for all $v \in V$ and $c \in C$. Thus, $C$ consists of homotheties, $D_{1} D_{2}$ also acts irreducibly on $V$, and $v_{\gamma}$ is $d_{2}$-dominant. Since $D_{1}$ commutes with $d_{2}$ we infer, as above, that $D_{1}$ preserves the direction of $v_{\gamma}$. Since $D_{1}$ is compact and connected, $v_{\gamma}$ is $D_{1}$-invariant. Since $D_{1}$ commutes with $C D_{2}$, the subspace of $D_{1}$-invariant vectors is preserved by the action of $C D_{1} D_{2}$. From the irreducibility of $\mathrm{Zc}_{0}(\Gamma)$, we conclude that $D_{1}=\mathrm{Id}$, hence $\mathrm{Zc}_{0}(\Gamma)=C D_{2}$.

Proof of Proposition 2.6. We consider the semigroup $\Gamma(S)$ generated by the finite set $S \subset \Gamma$. Clearly, if $S \subset S^{\prime}$, then $\Gamma(S) \subset \Gamma\left(S^{\prime}\right)$. We take a totally ordered family $S_{i}(i \in I)$ such that $\Gamma=\bigcup_{i \in I} \Gamma\left(S_{i}\right)$; we denote by $G_{0}^{i}$ the connected component of the identity in $\operatorname{Zc}\left(\Gamma\left(S_{i}\right)\right)$. Then, since $G_{0}^{i} \subset G_{0}^{j}$ if $S_{i} \subset S_{j}$, for some $\iota \in I$ we get

$$
H_{0}:=G_{0}^{\iota}=\bigcup_{i \in I} G_{0}^{i}=G_{0}^{k} \quad \text { if } S_{k} \supset S_{\iota} .
$$

We can suppose that $G_{0}^{i}=G_{0}^{\iota}$ for any $i \in I$. It follows that $H_{0}$ is normal in $\mathrm{Zc}\left(\Gamma\left(S_{i}\right)\right)$ for any $i \in I$, hence $H_{0}$ is normal in $\mathrm{Zc}(\Gamma)$. In particular, $H_{0} \subset$ $\mathrm{Zc}_{0}(\Gamma)$. We observe that $H_{0}$ has finite index in $\mathrm{Zc}\left(\Gamma\left(S_{i}\right)\right)$, hence $L=\mathrm{Zc}_{0}(\Gamma) /$ $H_{0}$ is an algebraic group which is the Zariski closure of the union of the finite subgroups $\Phi_{i}$ corresponding to $\mathrm{Zc}_{0}\left(\Gamma\left(S_{i}\right)\right)$. In view of Lemma 2.7 we know that the algebraic group $L$ has the same structure as $\mathrm{Zc}_{0}(\Gamma)$, in particular is reductive. We write it as the almost direct product of its connected center $C^{\prime} \subset \mathbb{R}^{*}$ and its commutator subgroup $D^{\prime}$. Passing to the factor group $L /$ $D^{\prime}$, using the finite subgroups $\Phi_{i}$, we get $C^{\prime}=\{\operatorname{Id}\}, L=D^{\prime}$. We consider a faithful, irreducible representation of the adjoint group of $L$ in a real vector space $V^{\prime}$. Then each finite subgroup $\Phi_{i}$ leaves invariant a positive definite quadratic form $q_{i}$. We can suppose that the forms $q_{i}$ are normalized and we denote by $q$ a cluster value of the $\left(q_{i}\right)_{i \in I}$. Then $q$ is invariant under the action of the topological closure $\Phi$ of $\bigcup_{i \in I} \Phi_{i}$. Since $\operatorname{Zc}\left(\bigcup_{i \in I} \Phi_{i}\right)=L=\operatorname{Zc}(\Phi)$, we see that $\Phi$ acts irreducibly on $V^{\prime}$, as $L$ itself. Since the kernel of $q$ is $\Phi$-invariant, it is trivial, hence $q$ is positive definite. It follows that $\Phi$ is compact. Since $\operatorname{Zc}(\Phi)=L$, we conclude that $L=\Phi$ is compact, hence from Lemma 2.7, $L=\{\mathrm{Id}\}$. It follows that $H_{0}=\mathrm{Zc}_{0}(\Gamma)=\mathrm{Zc}_{0}\left(\Gamma\left(S_{\iota}\right)\right)$. We can 
suppose that $\Gamma\left(S_{\iota}\right)$ contains a proximal element from $\Gamma \cap \mathrm{Zc}_{0}(\Gamma)$. Then $\Gamma\left(S_{\iota}\right)$ is finitely generated, and satisfies $\left(H_{2}\right)$. From Proposition 2.5 we see that condition $\left(H_{1}\right)$ is also satisfied by $\Gamma\left(S_{\iota}\right)$, since $\mathrm{Zc}_{0}\left(\Gamma\left(S_{\iota}\right)\right)=\mathrm{Zc}_{0}(\Gamma)$ acts irreducibly on $V$.

REMARK 2.8. We will see in Lemma 3.3 below that condition $\left(H_{0}\right)$ also remains valid after passing to a convenient finitely generated subsemigroup. However, in Proposition 2.6, this property cannot be achieved with condition $\left(H_{1}\right)$ alone. A simple counterexample is the following: suppose $\Gamma$ is the semigroup of rational rotations of the Euclidean plane, centered at the origin. Then any finitely generated subsemigroup $\Gamma^{\prime}$ preserves a regular polygon inscribed in the unit circle. Hence, condition $\left(H_{1}\right)$ is not satisfied by $\Gamma^{\prime}$.

This explains why we consider $\left(H_{1}\right)$ and $\left(H_{2}\right)$ simultaneously in Proposition 2.6.

3. Equivalence of the weak ID-property and ID-property. Let us recall the definitions of the weak ID-property and ID-property once again, in the context of subsemigroups of $\mathrm{M}_{\mathrm{inv}}(d, \mathbb{Z})$ acting in the usual way on $d$-dimensional tori. We say that a subsemigroup $\Gamma$ of $\mathrm{M}_{\text {inv }}(d, \mathbb{Z})$ has the $I D$-property if every infinite $\Gamma$-invariant subset of $\mathbb{T}^{d}$ is dense in $\mathbb{T}^{d}$. This is of course equivalent to the fact that every infinite closed $\Gamma$-invariant subset of $\mathbb{T}^{d}$ is $\mathbb{T}^{d}$ itself.

We say that a subsemigroup $\Gamma$ of $\mathrm{M}_{\mathrm{inv}}(d, \mathbb{Z})$ acting on the $d$-dimensional torus has the weak ID-property if for every $x \in \mathbb{T}^{d}$, the closure of the orbit $\overline{\Gamma x}$ is either finite or the whole $\mathbb{T}^{d}$.

Here it is convenient to use condition $\left(H_{0}\right)$ which is weaker than the hypothesis in Theorem 1.1.

Proposition 3.1. Let $\Gamma$ be a subsemigroup of $\mathrm{M}_{\mathrm{inv}}(d, \mathbb{Z})$ acting on $\mathbb{T}^{d}$ and satisfying condition $\left(H_{0}\right)$. Then $\Gamma$ has the weak ID-property if and only if $\Gamma$ has the ID-property.

To prove the above equivalence we need the following three lemmas.

Lemma 3.2. Suppose $S$ is a finite subset of $\mathrm{GL}(d, \mathbb{R})$ which generates a semigroup $\Gamma$ which satisfies $\left(H_{0}\right)$. Define

$$
C=\sup \{\|s\|: s \in S\} .
$$

Then for every $x \in V$ with $\|x\| \leq 1$ there exists an element $g \in \Gamma$ such that

$$
1<\|g x\| \leq C .
$$

Proof. Note that $C>1$, since $\Gamma$ is unbounded. We consider a sequence $s_{k} \in S$ such that the sequence $s_{n} \ldots s_{1} x,\|x\| \leq 1$, is unbounded, and define

$$
k=\sup \left\{n \in \mathbb{N}:\left\|s_{n} \ldots s_{1} x\right\| \leq 1\right\} .
$$


Then we have

$$
\left\|s_{k+1}\right\| \leq C, \quad\left\|s_{k} \ldots s_{1} x\right\| \leq 1, \quad\left\|s_{k+1} \ldots s_{1} x\right\|>1 .
$$

It follows that

$$
1<\left\|s_{k+1} s_{k} \ldots s_{1} x\right\| \leq C\left\|s_{k} \ldots s_{1} x\right\| \leq C .
$$

Then the conclusion follows with $g=s_{k+1} \ldots s_{1}$.

Lemma 3.3. Let $\Gamma$ be a subsemigroup of $\mathrm{GL}(d, \mathbb{R})$ which satisfies condition $\left(H_{0}\right)$. Then $\Gamma$ contains a finitely generated subsemigroup which satisfies $\left(H_{0}\right)$.

Proof. For any finite subset $S \subset \Gamma$, we denote by $\Gamma(S)$ the semigroup generated by $S$, and by $V(S)$ the subspace of vectors $v \in V$ such that $\Gamma(S) v$ is bounded. We observe that the inclusion $S \subset S^{\prime}$ implies $V\left(S^{\prime}\right) \subset V(S)$. We consider a totally ordered family of finite subsets $S_{\iota}(\iota \in I)$ such that $\Gamma=\bigcup_{\iota \in I} \Gamma\left(S_{\iota}\right)$. Then $W=\bigcap_{\iota \in I} V\left(S_{\iota}\right)$ is of the form $V\left(S_{j}\right)$ for some $j \in I$ and we have $V\left(S_{\iota}\right)=V\left(S_{j}\right)$ if $S_{\iota} \supset S_{j}$. It follows that $W$ is $\Gamma$-invariant. Furthermore, for any $v \in W$ and $\iota \in I, \Gamma\left(S_{\iota}\right) v$ is bounded.

We show that, if $W \neq\{0\}$, then $\Gamma v$ is bounded, for some $v \in W \backslash\{0\}$. Hence $W=\{0\}$ by condition $\left(H_{0}\right)$. This implies that condition $\left(H_{0}\right)$ is satisfied by $\Gamma\left(S_{j}\right)$.

We consider the complexified vector space $W^{\mathbb{C}} \subset V^{\mathbb{C}}$, a $\Gamma$-irreducible subspace $U \subset W^{\mathbb{C}}$, and the action of $\Gamma\left(S_{\iota}\right)$ on $U$. Since every $\Gamma\left(S_{\iota}\right)$-orbit in $W$ is bounded for any $\gamma \in \Gamma\left(S_{\iota}\right)$ we have $\left|\lambda_{\gamma}\right| \leq 1$, hence $|\operatorname{Tr} \gamma|_{U} \mid \leq \operatorname{dim} U$ for any $\gamma \in \Gamma$.

Since the action of $\Gamma$ on $U$ is irreducible, Burnside's theorem implies that the algebra $\operatorname{End}(U)$ is generated by $\Gamma$, i.e. there exist $\gamma_{1}, \ldots, \gamma_{r}$ in $\Gamma$ such that the linear forms $f_{1}, \ldots, f_{r}$ on $\operatorname{End}(U)$ defined by

$$
f_{k}(w)=\operatorname{Tr}\left(\gamma_{k} w\right) \quad(1 \leq k \leq r)
$$

form a basis of $(\operatorname{End}(U))^{*}$. Since $\left|f_{k}(\gamma)\right| \leq \operatorname{dim} U$ for every $\gamma \in \Gamma$, and the family $\left\{f_{k}\right\}$ forms a basis of $(\operatorname{End}(U))^{*}$, we deduce that $\left.\Gamma\right|_{U}$ is bounded. Then any $\Gamma$-orbit in $U$ is bounded. Hence the same is true for the conjugate space $\bar{U} \subset V^{\mathbb{C}}$, and for the sum $U+\bar{U} \subset V^{\mathbb{C}}$. In particular, any $v \in(U+\bar{U}) \cap V$ has a bounded $\Gamma$-orbit. Hence from condition $\left(H_{0}\right),(U+\bar{U}) \cap V=\{0\}$, $U=\{0\}, W=\{0\}$.

Let $B_{\varepsilon} \subset \mathbb{R}^{d}$ denote the ball with radius $\varepsilon$ and center 0 . For $\varepsilon<1 / 2$, we also denote by $B_{\varepsilon}$ the homeomorphic image of $B_{\varepsilon} \subset \mathbb{R}^{d}$ under the canonical quotient map $p: \mathbb{R}^{d} \rightarrow \mathbb{T}^{d}=\mathbb{R}^{d} / \mathbb{Z}^{d}$.

Lemma 3.4. Let $\Gamma$ be a subsemigroup of $\mathrm{M}_{\mathrm{inv}}(d, \mathbb{Z})$ which satisfies $\left(H_{0}\right)$. Then there exists $\varepsilon=\varepsilon_{\Gamma}>0$ such that for every $0 \neq x \in \mathbb{T}^{d}$,

$$
\Gamma x \cap \mathbb{T}^{d} \backslash B_{\varepsilon} \neq\{0\} .
$$


Proof. From Lemmas 3.3 and 3.2 above we can find $C>1$ such that for any $x \in B_{\varepsilon} \subset \mathbb{R}^{d}, \varepsilon<1 / 2$, there exists $g \in \Gamma$ such that

$$
\varepsilon \leq\|g x\| \leq C \varepsilon .
$$

If $\varepsilon_{\Gamma}=1 / 2 C<1 / 2$ we see that $\Gamma x \not \subset B_{\varepsilon}$ for every $x \in B_{\varepsilon} \subset \mathbb{T}^{d}$. If for some $y \notin B_{\varepsilon}$ we had $\Gamma y \subset B_{\varepsilon}$, then $x=\gamma y \in B_{\varepsilon}$ for some $\gamma \in \Gamma$; hence, from the above observation, $\Gamma x \not \subset B_{\varepsilon}$; in particular, since $\Gamma x \subset \Gamma y$, we have $\Gamma y \not \subset B_{\varepsilon}$, and this is a contradiction.

Now we are ready to prove Proposition 3.1.

Proof of Proposition 3.1. It is obvious that the ID-property implies the weak ID-property. Therefore we have to prove the converse, i.e. any infinite closed subset $\Sigma$ with $\Gamma \Sigma \subset \Sigma$ is equal to $\mathbb{T}^{d}$.

If $\Gamma x$ is infinite for some $x \in \Sigma$, then the hypothesis implies $\overline{\Gamma x}=\mathbb{T}^{d}=\Sigma$. Hence we can suppose that $\Sigma$ is infinite, $\Sigma=\bigcup_{x \in \Sigma} \Gamma x$ and each $\Gamma x$ with $x \in \Sigma$ is finite. It follows that $\Sigma \subset p\left(\mathbb{Q}^{d}\right)$, hence $\Sigma$ is countable.

Now consider the sequence of derived sets,

$$
\Sigma^{0}=\Sigma \supset \Sigma^{1} \supset \cdots \supset \Sigma^{n} \supset \cdots,
$$

that is, $\Sigma^{n+1}$ is the set of limit points of $\Sigma^{n}$. Actually, the sequence (3.5) terminates, i.e. there is an index $n$ such that $\Sigma^{n}=\emptyset$. If not we consider $\Sigma^{\infty}:=\bigcap_{n=0}^{\infty} \Sigma^{n}$. Clearly, $\Sigma^{\infty}$ is a closed countable set such that the set $\left(\Sigma^{\infty}\right)^{\text {ac }}$ of limit points of $\Sigma^{\infty}$ is equal to $\Sigma^{\infty}$. This means that $\Sigma^{\infty}$ is a non-void and countable perfect set. Since every point of $\Sigma^{\infty}$ is closed and has empty interior in $\Sigma^{\infty}$, the Baire theorem says that $\Sigma^{\infty}$ also has empty interior in $\Sigma^{\infty}$, which is impossible. Therefore, there is $n \in \mathbb{N}$ such that

$$
\Sigma^{0}=\Sigma \supset \Sigma^{1} \supset \cdots \supset \Sigma^{n}=\emptyset .
$$

Without loss of generality we may assume $n=2$. It follows that $\Sigma^{1}$ is finite. In fact, otherwise $\Sigma^{2}$ would not be an empty set. Let $\left\{x_{1}, \ldots, x_{n}\right\}=\Sigma^{1} \subset$ $p\left(\mathbb{Q}^{d}\right)$ be the set of limit points of $\Sigma$ and let $q$ be a common denominator of $x_{i}, 1 \leq i \leq n$. Then $0 \in q \Sigma^{1}$ is the unique limit point of $q \Sigma$. Consider a ball $B_{\varepsilon}$ around 0 with $\varepsilon<\varepsilon_{\Gamma}$ given by Lemma 3.4. Then the points of $q \Sigma$ outside $B_{\varepsilon}$ have no limit point, hence form a finite set $F$. Now we can consider the $\Gamma$-orbits of these points, i.e. $\Gamma x, x \in F$. They form a $\Gamma$-invariant finite set $F^{\prime}=\bigcup_{x \in F} \Gamma x$ that we can exclude from $q \Sigma$ without changing its properties. Therefore, now we have the new set $\Sigma^{\prime}=q \Sigma \backslash F^{\prime}$ which is closed, infinite, $\Gamma$-invariant and fully included in $B_{\varepsilon}$, and this contradicts Lemma 3.4.

4. Dominant vectors, a cohomological equation and the spectrum of $\Gamma$. As in Section 2, $\Gamma$ is a subsemigroup of $\mathrm{GL}(V)=\mathrm{GL}(d, \mathbb{R})$ and we consider its action on $V$ and $\mathbb{P}(V)=\pi(V \backslash\{0\})$. 
We define the asymptotic sets:

$$
\begin{aligned}
& L_{\Gamma}=\overline{\left\{\pi\left(v_{0}\right): v_{0} \text { is a dominant vector for } \Gamma\right\}}, \\
& \widetilde{L}_{\Gamma}=\left\{v \neq 0: \pi(v) \in L_{\Gamma}\right\}=\pi^{-1}\left(L_{\Gamma}\right) .
\end{aligned}
$$

We start with the following proposition which is a semigroup version of a result of Guivarc'h and Conze (cf. [6, Proposition 3.2]).

Proposition 4.1. Let $\Sigma$ be a $\Gamma$-invariant subset of $V \backslash\{0\}$ such that $0 \in \bar{\Sigma}$. Then, under assumptions $\left(H_{0}\right),\left(H_{1}\right)$ and $\left(H_{2}\right)$, for any proximal and quasi-expanding element $\gamma \in \Gamma$ there exists a $\gamma$-dominant vector $u_{0}$ such that

$$
\gamma^{\mathbb{Z}} u_{0}:=\left\{\gamma^{k} u_{0}: k \in \mathbb{Z}\right\} \subset \bar{\Sigma} .
$$

Proof. Let $V=V_{\gamma}^{\max } \oplus V_{\gamma}^{<}$be the decomposition of $V$ relative to a proximal and quasi-expanding element $\gamma \in \Gamma$.

Let $x_{i} \in \Sigma$ and $x_{i} \rightarrow 0$ as $i \rightarrow \infty$. Then there exists a sequence $\left\{\alpha_{i}\right\}$ of reals and $w \in V$ such that $\alpha_{i} x_{i} \rightarrow w$ as $i \rightarrow \infty$. We will show that without loss of generality we can assume that $w \notin V_{\gamma}^{<}$. In fact, since $\Gamma$ acts strongly irreducibly on $V$, by Lemma 2.2 one can find an element $h \in \Gamma$ such that $h w \notin V_{\gamma}^{<}$, i.e. $w \notin h^{-1} V_{\gamma}^{<}$. Define

$$
\Gamma_{1}=h^{-1} \Gamma h, \quad \gamma_{1}=h^{-1} \gamma h \in \Gamma_{1}, \quad \Sigma_{1}=h^{-1} \Sigma .
$$

Then $\gamma_{1}$ is proximal in $\Gamma_{1}, w \notin h^{-1} V_{\gamma}^{<}=V_{\gamma_{1}}^{<}$, and $\Sigma_{1}$ is a $\Gamma_{1}$-invariant subset that contains 0 as a limit point. Assume that we have found a non-zero vector $u_{0} \in V_{\gamma_{1}}^{\max }$ such that $\gamma_{1}^{\mathbb{Z}} u_{0} \subset \bar{\Sigma}_{1}$. Then $h^{-1} \gamma^{\mathbb{Z}} h u_{0} \subset h^{-1} \bar{\Sigma}$, i.e., $\gamma^{\mathbb{Z}} h u_{0} \subset \bar{\Sigma}$. But $h u_{0} \in V_{\gamma}^{\max }$ and we are done.

Thus from the very beginning we can assume that $w \notin V_{\gamma}^{<}$.

Let $e_{1}, \ldots, e_{n}$ be a basis of $V$ such that $e_{1} \in V_{\gamma}^{\max },\left\|e_{1}\right\|=1$ and $e_{2}, \ldots, e_{n} \in V_{\gamma}^{<}$. Let $\phi_{j}: V \rightarrow \mathbb{R}$ be linear forms such that

$$
x=\sum_{j=1}^{n} \phi_{j}(x) e_{j}, \quad x \in V .
$$

Let $\Phi: V=V_{\gamma}^{\max } \oplus V_{\gamma}^{<} \rightarrow V_{\gamma}^{\max }$ be the projection along $V_{\gamma}^{<}$, i.e., $\Phi(x)=$ $\phi_{1}(x) e_{1}$. Since $w \notin V_{\gamma}^{<}$, it follows without loss of generality that $\Phi\left(x_{i}\right) \neq 0$ (since $\alpha_{i} x_{i} \rightarrow w \notin V_{\gamma}^{<}$). Since $\left|\lambda_{\gamma}\right|=\lambda>1$, there exists a sequence $\left\{p_{i}\right\}$ of integers such that $p_{i} \rightarrow \infty$ and

$$
1 \leq \lambda^{p_{i}}\left|\phi_{1}\left(x_{i}\right)\right| \leq \lambda .
$$

Now passing to a subsequence if necessary one can find a $\gamma$-dominant vector $u_{0} \in V_{\gamma}^{\max }$ such that $\gamma^{p_{i}}\left(\Phi\left(x_{i}\right)\right) \rightarrow u_{0}$ as $i \rightarrow \infty$. We will prove that $\gamma^{p_{i}}\left(x_{i}\right) \rightarrow u_{0}$ as $i \rightarrow \infty$.

Clearly, it is enough to show that

$$
\phi_{j}\left(x_{i}\right) \gamma^{p_{i}}\left(e_{j}\right) \rightarrow 0 \quad \text { as } i \rightarrow \infty \text {, for any } j>1 \text {. }
$$


In fact, $\gamma^{p_{i}}\left(x_{i}\right) \rightarrow u_{0}$ if $\gamma^{p_{i}}\left(\sum_{j=1}^{n} \phi_{j}\left(x_{i}\right) e_{j}\right)=\sum_{j=1}^{n} \phi_{j}\left(x_{i}\right) \gamma^{p_{i}}\left(e_{j}\right) \rightarrow u_{0}$ and so we are led to (4.3).

Therefore, we are going to prove (4.3). One has

$$
\phi_{j}\left(x_{i}\right) \gamma^{p_{i}}\left(e_{j}\right)=\frac{\phi_{j}\left(x_{i}\right)}{\left|\phi_{1}\left(x_{i}\right)\right|} \frac{\gamma^{p_{i}}\left(e_{j}\right)}{\lambda^{p_{i}}}\left\|\gamma^{p_{i}}\left(\Phi\left(x_{i}\right)\right)\right\|,
$$

where the first fraction tends to $\phi_{j}(w) /\left|\phi_{1}(w)\right|$, the second one tends to zero, and the third term tends to $\left\|u_{0}\right\|$ as $i \rightarrow \infty$.

Since $x_{i} \in \Sigma$ and $\gamma^{p_{i}} \in \Gamma$ for every $i \in \mathbb{N}$ it follows that $u_{0} \in \bar{\Sigma}$. Since $\lim _{i} \gamma^{p_{i}}\left(x_{i}\right)=u_{0}$, we infer that $\gamma^{-m}\left(u_{0}\right)=\lim _{i} \gamma^{p_{i}-m}\left(x_{i}\right)$. We see that $\gamma^{p_{i}-m}\left(x_{i}\right) \in \Sigma$ for almost all $i$, thus $\gamma^{-m} u_{0} \in \bar{\Sigma}$.

REMARK 4.4. Notice that the condition (4.2) implies that $0 \in \bar{\Sigma}$. In fact, simply take a sequence $\mathbb{Z} \ni k_{n}$ such that $k_{n} \rightarrow-\infty$.

Proposition 4.5. Under conditions $\left(H_{1}\right)$ and $\left(H_{2}\right)$, the set $L_{\Gamma}$ is the unique minimal $\Gamma$-invariant closed subset of $\mathbb{P}(V)$.

Proof. We first show that $L_{\Gamma}$ is $\Gamma$-invariant. Consider $g \in \Delta_{\Gamma}$, and $u=\lim _{n} g^{2 n} /\left\|g^{2 n}\right\|$, where the limit exists in $\operatorname{End}(V)$ by proximality of $g$. Consider a decomposition $V=V_{g}^{<} \oplus V_{g}^{\max }$. Then $u$ is a multiple of the projection of $V$ onto $V_{g}^{\max }$ along $V_{g}^{<}$.

On the other hand, we consider $\gamma \in \Gamma$ and want to show that $\gamma \cdot g^{+} \in L_{\Gamma}$. We observe that for any $\delta \in \Gamma$, we have

$$
\lim _{n}\left\|\gamma \frac{g^{2 n}}{\left\|g^{2 n}\right\|} \delta-\gamma u \delta\right\|=0
$$

We have $\operatorname{Im}(\gamma u \delta)=\gamma(\operatorname{Im} u)$ and $\operatorname{Ker}(\gamma u \delta)=\delta^{-1}(\operatorname{Ker} u)$. We note that $\gamma u \delta$ has rank one, like $u$, hence $\gamma u \delta$ will be a multiple of a one-dimensional projection if $\gamma(\operatorname{Im} u) \not \subset \delta^{-1}(\operatorname{Ker} u)$, i.e. $\delta(\operatorname{Im}(\gamma u)) \not \subset \operatorname{Ker} u$.

Since $\Gamma$ is strongly irreducible, Lemma 2.2 shows that such a $\delta \in \Gamma$ exists. Then, as in the proof of Proposition 2.3, perturbation theory shows that for $n$ large, $\gamma\left(g^{2 n} /\left\|g^{2 n}\right\|\right) \delta$ has a simple dominant eigenvalue and the corresponding eigenvector is close to $\gamma\left(v_{g}\right)$. In other words,

$$
\gamma g^{2 m} \delta \in \Delta_{\Gamma}, \quad \gamma \cdot g^{+}=\lim _{m}\left(\gamma g^{2 m} \delta\right)^{+} .
$$

Hence $\gamma L_{\Gamma} \subset L_{\Gamma}$.

Now let $\Lambda$ be a closed $\Gamma$-invariant subset of $\mathbb{P}(V)$ and let us show that $\Lambda \supset L_{\Gamma}$. Since $\Gamma$ is strongly irreducible, $\Lambda$ is not contained in a proper subspace. In particular $\Lambda \not \subset V_{\gamma}^{<}$, hence there exists $x \in \Lambda$ with $x \notin V_{\gamma}^{<}$. Then $\gamma^{+}=\lim _{n} \gamma^{n} . x \in \Lambda$. Since $\Lambda$ is closed, we have $L_{\Gamma} \subset \Lambda$. This shows that $L_{\Gamma}$ is minimal and is the unique minimal subset of $\mathbb{P}(V)$.

Proposition 4.6 (Proposition 2.2 in [7]). Let $\Gamma$ be a subsemigroup of $\mathrm{GL}(d, \mathbb{R}), d>1$, satisfying condition $\left(H_{1}\right)$ and $\left(H_{2}\right)$. Let $S$ be a generating 
subset of $\Gamma$. If $\varphi$ is a non-zero, continuous function on $\mathbb{P}^{d-1}, t$ is real and $\theta \in[0,2 \pi)$, then the equation

$$
\forall \gamma \in S, \forall x \in L_{\Gamma}, \quad \varphi(\gamma \cdot x)\|\gamma x\|^{i t}=e^{i \theta} \varphi(x)
$$

has no solution, unless $\theta=0, t=0, \varphi(x) \equiv$ const on $L_{\Gamma}$.

Proof. Clearly we can suppose that $|\operatorname{det} \gamma|=1$ for $\gamma \in \Gamma$. Consider the function $\psi(v)$ defined on $\widetilde{L}_{\Gamma}$ by the formula $\psi(v)=\varphi(\pi(v))\|v\|^{i t}$. Then the relation for $\varphi$ can be written as

$$
\forall \gamma \in S, \quad \psi(\gamma v)=e^{i \theta} \psi(v) .
$$

Suppose that $t \neq 0$ and put $\log \varrho=2 \pi /|t|$. Then additionally we have $\psi\left( \pm \varrho^{k} v\right)=\psi(v)$ and the condition $\psi(\lambda v)=\psi(v)$ for some $v \in \widetilde{L}_{\Gamma}$ and some $\lambda \in \mathbb{R}_{+}^{*}$ implies that $\lambda= \pm \varrho^{k}$, where $k \in \mathbb{Z}$.

Let $c$ be any of the values of $\psi$ and put $L_{c}=\psi^{-1}(\{c\}) \subset \widetilde{L}_{\Gamma}$. Then, since $\psi$ is continuous, $L_{c}$ is a non-empty closed subset of $\widetilde{L}_{\Gamma}$ which satisfies

$$
\forall \gamma \in S, \quad \gamma\left(L_{c}\right) \subset L_{c e^{i \theta}} .
$$

For every $\lambda \in H_{\varrho}$, which is the group of homotheties of the form $\pm \varrho^{k}, k \in \mathbb{Z}$, we also have

$$
\lambda L_{c}=L_{c} .
$$

If now $u \in \operatorname{End}\left(\mathbb{R}^{d}\right)$ satisfies $u=\lim _{k} \varrho^{-n_{k}} \gamma_{k}$ with $\gamma_{k} \in \Gamma, \varrho^{-1} \leq\|u\|<1$, then we have $|\operatorname{det} u|=\lim _{k} \varrho^{-n_{k} d}=0$ and $u\left(L_{c}\right) \subset L_{c e^{i \alpha}} \cup\{0\}$ with $\alpha \in \mathbb{R}$. From condition $\left(H_{2}\right)$ we can choose $\gamma_{k}=\gamma^{k} \in \Gamma$ with $W=\operatorname{Ker} u \neq 0$ having codimension 1 . Since $\operatorname{Im} u=\mathbb{R} v_{\gamma}=\mathbb{R} a, a \neq 0$, we find that $u\left(L_{c}\right) \subset$ $H_{\varrho} a \cup\{0\}$. Since $u^{-1}(a)=b+W$ with $b \in \mathbb{R}^{d} \backslash\{0\}$ we deduce that $L_{c} \subset$ $W \cup H_{\varrho}(b+W)$. It follows that, in the quotient space $\mathbb{R}^{d} / W, L_{c}$ is projected onto a set which is countable and $H_{\varrho}$-invariant. If $W_{i}, 1 \leq i \leq r$, is a family of such subspaces then $\bigcap_{i=1}^{r} W_{i}$ has the same property. In fact $V / \bigcap_{i=1}^{r} W_{i}$ can be identified with the diagonal subspace of $V / W_{1} \times \cdots \times V / W_{r}$, and so the projection of $L_{c}$ into $V / \bigcap_{i=1}^{r} W_{i}$ can be identified with a subset of the product of the projections of $L_{c}$. Hence, such a projection is countable and $H_{\varrho}$-invariant. Since the intersection of any family of subspaces with the above properties is a finite intersection, there exists a unique minimal subspace $W_{0}$ which has these properties. This subspace is unchanged when $c$ is replaced by $c e^{i \alpha}$. As a consequence, the condition $\gamma\left(L_{c}\right) \subset L_{c e^{i \theta}}$ for $\gamma \in \Gamma$ implies that $\gamma\left(W_{0}\right)=W_{0}$. Since $\pi\left(L_{c}\right)=L_{\Gamma}$ and $L_{\Gamma}$ is uncountable (see Lemma 5.1), $W_{0}$ is proper. This contradicts the irreducibility of $\Gamma$, and so $t=0, e^{-i \theta} \varphi(\gamma \cdot x)=\varphi(x)$ for all $x \in L_{\Gamma}$ and $\gamma \in S$. Therefore, for every $n$, all $\gamma_{i} \in S$, and all $x \in L_{\Gamma}$ we have $e^{-i n \theta} \varphi\left(\gamma_{1} \ldots \gamma_{n} . x\right)=\varphi(x)$. Since $\Gamma=\bigcup_{n=0}^{\infty} S^{n}$ satisfies $\left(H_{2}\right)$, we deduce that $\varphi \equiv$ const on $L_{\Gamma}$, and $e^{i \theta}=1$. In fact, suppose that there are two different points $x$ and $y$ in $L_{\Gamma}$ such that 
$\varphi(x) \neq \varphi(y)$. Then

$$
\varphi(x)=e^{-i n \theta} \varphi\left(\gamma_{1} \ldots \gamma_{n} . x\right) \neq e^{-i n \theta} \varphi\left(\gamma_{1} \ldots \gamma_{n} . y\right)=\varphi(y)
$$

and so,

$$
0<|\varphi(x)-\varphi(y)|=\left|\varphi\left(\gamma_{1} \ldots \gamma_{n} . x\right)-\varphi\left(\gamma_{1} \ldots \gamma_{n} . y\right)\right|
$$

for every $n \in \mathbb{N}$ and all $\gamma_{i} \in S$. By proximality of $\Gamma$ on $\mathbb{P}(V)$ (see Proposition 2.3) there exists a sequence $\left\{\gamma_{i}\right\}_{i=1}^{\infty}$ such that

$$
\lim _{n} \delta\left(\gamma_{1} \ldots \gamma_{n} . x, \gamma_{1} \ldots \gamma_{n} . y\right)=0
$$

By continuity of $\varphi$ we a get a contradiction to (4.7).

Now we consider the set $S_{\Gamma}=\left\{\log \left|\lambda_{g}\right|: g \in \Delta_{\Gamma}\right\}$, the so-called spectrum of $\Gamma([8])$.

The following corollary, which is a complement to Proposition 4.6 above, clarifies the connections between the aperiodicity hypotheses on $\Gamma$ considered by Kesten in [22], Guivarc'h and Raugi in [17] (Proposition 3 and Lemma p. 45), Lalley in [23] (Corollaries 11.3, 11.4) and the geometric conditions considered by Eberlein in [11] and Dal'bo in [8] in the context of lengths of closed geodesics in the case of negative curvature. For an extension of these results and their use in the more general setting of semisimple groups see [3] and [19]. The corollary below also explains why aperiodicity conditions are not explicitly stated in Theorem 1.1, as in [4] and [26].

Corollary 4.8. Suppose $\Gamma \subset \mathrm{GL}(d, \mathbb{R})$ is a subsemigroup which satisfies $\left(H_{1}\right),\left(H_{2}\right)$ and define $S_{\Gamma}=\left\{\log \left|\lambda_{g}\right|: g \in \Delta_{\Gamma}\right\}$. Then $S_{\Gamma}$ generates a dense subgroup of $\mathbb{R}$.

For the proof, which uses standard arguments of thermodynamic formalism, we need three lemmas, the first of them being well known (see [5, pp. 90-94]).

Lemma 4.9. Let $A$ be a finite set, $\Omega$ the compact metric space $A^{\mathbb{N}}$, and $\theta$ the shift transformation on $\Omega$ given by $(\theta \omega)_{k}=\omega_{k+1}, k \in \mathbb{N}$. For a function $\varphi$ on $\Omega$ define

$$
S_{n} \varphi(\omega)=\sum_{k=0}^{n-1} \varphi \circ \theta^{k}(\omega) .
$$

Suppose $\varphi$ is Hölder continuous, and for any periodic point $\omega$ of period $p$, the sum $S_{p} \varphi(\omega)$ belongs to $\mathbb{Z}$. Then there exists a Hölder $\mathbb{Z}$-valued function $\varphi^{\prime}$ on $\Omega$ and a Hölder function $\psi$ such that $\varphi=\varphi^{\prime}+\psi-\psi \circ \theta$.

Lemma 4.10. Suppose $g, h \in \mathrm{GL}(d, \mathbb{R})$ are such that $h$ is proximal and g. $h^{+} \notin V_{h}^{<}$. Then, for $n=2 p$ large, $g h^{n}$ is proximal and

$$
\lim _{n}\left(g h^{n}\right)^{+}=g \cdot h^{+}, \quad \lim _{n} V_{g h^{n}}^{<}=V_{h}^{<} .
$$


Proof. We consider the sequence of linear maps $u_{n}=h^{n} /\left\|h^{n}\right\|$ and observe that $u_{n}$ converges towards a map $\pi_{h}$ which is proportional to the projection on $\mathbb{R} v_{h}$ along the subspace $V_{h}^{<}$. Hence $g u_{n}$ converges towards $g \pi_{h}$. We have

$$
\operatorname{Im}\left(g \pi_{h}\right)=\mathbb{R} g \cdot h^{+}, \quad \operatorname{Ker}\left(g \pi_{h}\right)=V_{h}^{<} .
$$

Hence, if $g . h^{+} \notin V_{h}^{<}$, then $g \pi_{h}$ is collinear to a projection onto a one-dimensional subspace. Since $g \pi_{h}$ has a simple dominant eigenvalue, the same is true for $g u_{n}$ for $n$ large. Therefore, for $n$ large, $g u_{n}$ is proximal, and moreover, we have the required convergence.

Lemma 4.11. Suppose $\Gamma \subset \mathrm{GL}(V)$ is a subsemigroup and satisfies conditions $\left(H_{1}\right),\left(H_{2}\right)$. Then there exist $a, b \in \Delta_{\Gamma}$ such that $a^{+} \neq b^{+}, V_{a}^{<} \neq V_{b}^{<}$ and $a^{+} \notin V_{b}^{<}, b^{+} \notin V_{a}^{<}$.

Proof. We consider the transposed semigroup $\Gamma^{t}$ acting on the dual space $V^{*}$ and the projective space $\mathbb{P}\left(V^{*}\right)$. From Remark 2.1(iii) conditions $\left(H_{1}\right)$ and $\left(H_{2}\right)$ remain valid for $\Gamma^{t}$ and we can consider the corresponding asymptotic set $L_{\Gamma^{t}}=L_{\Gamma}^{*}$. We fix $a \in \Delta_{\Gamma}$ and observe that we can find $b \in \Delta_{\Gamma}$ such that $V_{b}^{<} \neq V_{a}^{<}, a^{+} \notin V_{b}^{<}$. Otherwise there would be a dense subset of $L_{\Gamma}^{*}$ contained in the union of the two projective subspaces defined by $V_{a}^{<}$and $a^{+}$in $\mathbb{P}\left(V^{*}\right)$. Hence $L_{\Gamma}^{*}$ itself would be contained in such a union. But, from Lemma 5.1 below, this is impossible. If $b^{+} \notin a^{+} \cup V_{a}^{<}$, we have found the required pair $(a, b)$. If not, we consider $g \in \Gamma$ and the sequence $g b^{n}, n \in 2 \mathbb{N}$. In view of Lemma 2.2 we can choose $g \in \Gamma$ such that

$$
g . b^{+} \notin V_{b}^{<} \cup V_{a}^{<} \cup a^{+} .
$$

Then we can apply Lemma 4.10 and replace $b$ by $g b^{n}=b^{\prime}$ for $n$ large. Under this condition, $V_{b^{\prime}}^{<}$is close to $V_{b}^{<}$and the relations are still satisfied. Since $\left(b^{\prime}\right)^{+}$is close to $g \cdot b^{+}$and $g \cdot b^{+} \notin a^{+} \cup V_{a}^{<}$, the condition $\left(b^{\prime}\right)^{+} \notin a^{+} \cup V_{a}^{<}$is also satisfied. Hence, we can take $\left(a, b^{\prime}\right)$ as the required pair.

Proof of Corollary 4.8. Since $\Gamma$ satisfies $\left(H_{1}\right)$ and $\left(H_{2}\right)$ we can choose $a_{1}, a_{2}$ in $\Gamma$ according to Lemma 4.11. Let $C_{1}, C_{2}$ be closed and disjoint neighborhoods of $a_{1}^{+}, a_{2}^{+}$in $\mathbb{P}(V)$ such that $\left(C_{1} \cup C_{2}\right) \cap\left(V_{a_{1}}^{<} \cup V_{a_{2}}^{<}\right)=\emptyset$, and let $o$ be a point outside $V_{a_{1}}^{<} \cup V_{a_{2}}^{<} \cup C_{1} \cup C_{2}$. Then, for $i=1,2$,

$$
\lim _{n} a_{i}^{n} \cdot\left(C_{1} \cup C_{2}\right)=a_{i}^{+}, \quad \lim _{n} a_{i}^{n} \cdot o=a_{i}^{+} .
$$

If we take $n$ large and set $a=a_{1}^{n}, b=a_{2}^{n}$, we have

$$
\text { a.o } \in C_{1} \text {, b.o } \in C_{2} \text {, a. }\left(C_{1} \cup C_{2}\right) \subset \operatorname{Int} C_{1}, b .\left(C_{1} \cup C_{2}\right) \subset \operatorname{Int} C_{2} \text {. }
$$

It follows from (4.12) that the semigroup $\Gamma(a, b)$ generated by $a, b$ is free. In order to prove Corollary 4.8 we can suppose $\Gamma=\Gamma(a, b)$. We consider the trivial metric $\delta$ on $\{a, b\}$ and endow $\Omega=\{a, b\}^{\mathbb{N}}$ with the metric $\delta\left(\omega, \omega^{\prime}\right)=$ $\sum_{k=1}^{\infty} 2^{-k} \delta\left(\omega_{k}, \omega_{k}^{\prime}\right)$. We define a homeomorphism $\mathfrak{z}$ between $\Omega$ and $L_{\Gamma}$ as 
follows. We observe that if $\omega=\left(a_{k}\right)_{k \in \mathbb{N}}$ and $a_{k} \in\{a, b\}$, then it follows from (4.12) that the sequence $a_{1} \ldots a_{n} . o$ converges to $\mathfrak{z}(\omega) \in C_{1} \cup C_{2}$. It is easy to verify that $\mathfrak{z}$ is a bi-Hölder homeomorphism, hence we can transfer properties of $(\Omega, \theta)$ to the action of $\Gamma$ on $L_{\Gamma}$. We consider $\mathfrak{z}(\omega)$ as a unit vector in $V$ and observe that, by definition of $\mathfrak{z}$,

$$
a_{1}(\omega) \mathfrak{z}(\theta \omega)=\varepsilon(\omega)\left\|a_{1}(\omega) \mathfrak{z}(\theta \omega)\right\|_{\mathfrak{z}}(\omega),
$$

with $\varepsilon(\omega)=+1$ or -1 . It follows that, if we set

$$
\varphi(\omega)=\log \left\|a_{1}(\omega) \mathfrak{z}(\theta \omega)\right\|, \quad S_{n} \varphi(\omega)=\sum_{k=0}^{n-1} \varphi\left(\theta^{k} \omega\right),
$$

we have, with $\gamma=a_{1} \ldots a_{n-1} \in \Gamma$ and $x=\mathfrak{z}\left(\theta^{n} \omega\right) \in L_{\Gamma}$,

$$
S_{n} \varphi(\omega)=\log \|\gamma x\| \text {. }
$$

Given a Hölder function $\psi$ on $\Omega$ we define a Hölder function $\bar{\psi}$ on $L_{\Gamma}$ by $\bar{\psi}[\mathfrak{z}(\omega)]=\psi(\omega)$ and we also have $\psi(\omega)-\psi\left(\theta^{n} \omega\right)=\bar{\psi}(\gamma \cdot x)-\bar{\psi}(x)$. In particular, if $\omega \in \Omega$ is periodic with period $p\left(\theta^{p} \omega=\omega\right)$, then $\mathfrak{z}(\omega)$ is a dominant eigenvector of $\gamma=a_{1} \ldots a_{p-1}$ and the corresponding eigenvalue $\lambda_{\gamma}$ satisfies

$$
\log \left|\lambda_{\gamma}\right|=S_{p} \varphi(\omega) .
$$

If $S_{\Gamma}$ does not generate a dense subgroup of $\mathbb{R}$, then for some positive $c$ we have $S_{\Gamma} \subset c \mathbb{Z}$, hence $S_{p} \varphi(\omega) \in c \mathbb{Z}$ for any periodic point $\omega$ and we can apply Lemma 4.9 to the function $(1 / c) \varphi$. In particular, the function $e^{2 i \pi \varphi / c}$ can be written in the form $e^{2 i \pi(\psi-\psi \circ \theta)}$, where $\psi$ is a Hölder function on $\Omega$. We can define $\bar{\psi}$ on $L_{\Gamma}$ as above and write $u(x)=e^{2 i \pi \bar{\psi}}$. Then $u$ is continuous and we obtain, with $\gamma=a_{1} \ldots a_{n}, x=\mathfrak{z}\left(\theta^{n} \omega\right)$,

$$
\|\gamma x\|^{2 i \pi / c}=\frac{u(\gamma \cdot x)}{u(x)} .
$$

We extend $u$ to $\mathbb{P}(V)$ as a continuous function, again denoted by $u$. Then we have

$$
\forall x \in L_{\Gamma}, \forall \gamma \in \Gamma, \quad\|\gamma x\|^{2 i \pi / c}=\frac{u(\gamma \cdot x)}{u(x)} .
$$

In view of Proposition 4.6, this implies $2 i \pi / c=0, u=1$, and this is impossible.

5. Random walks on a vector space and its factor spaces. In this section, relying strongly on [13], [17] (see also [18]), we develop the random walk approach to the study of $\Gamma$-orbits on $V \backslash\{0\}$ and other related $\Gamma$-spaces. The main new results are Theorems 5.10 and 5.19 and their corollaries. They give Theorems 1.3 and 1.2 of the Introduction. In particular, Corollary 5.22 is one of the main tools for the study of $\Gamma$-orbits on the torus $\mathbb{T}^{d}$ if $\Gamma \subset \mathrm{M}_{\text {inv }}(d, \mathbb{Z})$, i.e., for Theorem 1.1. 
Let $\mu$ be a probability measure on $G=\mathrm{GL}(V)$, and $\Gamma_{\mu}$ the closed subsemigroup generated by the support $S_{\mu}$ of $\mu$. We denote by $M^{1}(X)$ the set of probability measures on a given Polish space $X$. We set $\Omega=S_{\mu}^{\mathbb{N}}$ and we consider the probability measure $\mathbf{P}_{\mu}=\mu^{\otimes \mathbb{N}}$ on $\Omega$; the shift $\theta$ on $\Omega$ given by $(\theta \omega)_{k}=\omega_{k+1}(k \in \mathbb{N})$ preserves $\mathbf{P}_{\mu}$ and the components $\omega_{k}=g_{k}(\omega)$ of $\omega$ are i.i.d. $G$-valued random variables of law $\mu$. From the Markov-Kakutani theorem, there exists a probability measure $\nu$ on $\mathbb{P}(V)$ which is $\mu$-stationary, i.e.,

$$
\mu * \nu=\int g . \nu d \mu(g)=\nu .
$$

We are going to establish that $[\mathbb{P}(V), \nu]$ is a $\mu$-boundary (see [13]), i.e.,

$$
\lim _{n} g_{1} g_{2} \ldots g_{n} \cdot \nu=\delta_{z_{\omega}},
$$

where $z_{\omega} \in \mathbb{P}(V)$. This will allow us to derive some properties of the typical sequences

$$
S_{n}=g_{n} g_{n-1} \ldots g_{1} \quad \text { and } \quad X_{n}=g_{1} g_{2} \ldots g_{n},
$$

and of the transposed maps $S_{n}^{t}$ and $X_{n}^{t}$.

Lemma 5.1. Assume that $\Gamma=\Gamma_{\mu}$ satisfies condition $\left(H_{1}\right)$, and let $\nu$ be a $\mu$-stationary measure. Then $\nu$ gives zero mass to every projective subspace. Furthermore, if $\Gamma$ also satisfies $\left(H_{2}\right)$, then $L_{\Gamma}$ is not contained in a countable union of subspaces.

Proof. Let $W$ be a projective subspace of minimal dimension such that $\nu(W)>0$. Define

$$
\sigma=\sum_{k \geq 1}\left(1 / 2^{k}\right) \mu^{* k}
$$

and consider the function $f(g)=g \cdot \nu(W)=\nu\left(g^{-1} \cdot W\right)$. This function is $\mu$-harmonic, i.e. satisfies

$$
\int f(g h) d \mu(h)=\int f(g h) d \sigma(h)=f(g),
$$

and reaches its maximum. In fact, the hypothesis on $W$ gives $\nu(g . W \cap$ $\left.g^{\prime} . W\right)=0$ if $g . W \neq g^{\prime} . W$, so the set of $g . W$ such that $\nu(g . W)>\delta$ is finite for every $\delta$. Then if $f\left(g_{0}\right)=\sup _{g \in G} f(g)$, the equation $f\left(g_{0}\right)=\int f\left(g_{0} h\right) d \sigma(h)$ gives $f\left(g_{0} h\right)=f\left(g_{0}\right), \sigma$-a.e. Let $E$ be the set of subspaces $W^{\prime}=g^{-1} . W$ such that $\nu\left(W^{\prime}\right)=f\left(g_{0}\right)$. Then, from above, $E$ is finite and $\Gamma^{-1}$-invariant. Hence the strong irreducibility of $\Gamma$ gives a contradiction.

If $\left(H_{2}\right)$ is also satisfied by $\Gamma$, then $L_{\Gamma}$ is well defined. From the MarkovKakutani theorem, we know that there exists a $\mu$-stationary measure $\lambda$ such that $\lambda\left(L_{\Gamma}\right)=1$, hence $S_{\lambda} \subset L_{\Gamma}$. Since $\lambda$ gives zero measure to every subspace, the same is true for a countable union of subspaces, hence $L_{\Gamma}$ cannot be contained in such a union. 
Proposition 5.3. Let $\nu$ be a $\mu$-stationary measure on $\mathbb{P}(V)$ and $\eta$ be as in (5.2). Then the sequence $g_{1} \ldots g_{n} . \nu$ converges $\mathbf{P}_{\mu}$-a.e. and for $\mathbf{P}_{\mu} \otimes \eta$-a.e. $(\omega, g) \in \Omega \times G$ we have

$$
\lim _{n} g_{1}(\omega) \ldots g_{n}(\omega) . \nu=\lim _{n} g_{1}(\omega) \ldots g_{n}(\omega) g . \nu .
$$

Proof. For a continuous function $\varphi$, we set $F_{\varphi}(g)=g . \nu(\varphi)$ and we observe that the relation $\int g . \nu d \mu(g)=\nu$ gives $\int F_{\varphi}(g h) d \mu(h)=F_{\varphi}(g)$, and consequently $F_{\varphi}\left(X_{n}\right)=g_{1} \ldots g_{n} . \nu(\varphi)$ is a bounded martingale. This martingale converges and, letting $\varphi$ vary in a dense countable part of $C(\mathbb{P}(V))$, we obtain the convergence of $g_{1} \ldots g_{n} . \nu$. In order to obtain the second claim, it suffices to show that $F_{\varphi}\left(X_{n} g\right)-F_{\varphi}\left(X_{n}\right)$ converges to zero $\mathbf{P}_{\mu} \otimes \mu^{* r}$-a.e. for every $r \geq 1$. But

$$
\int\left|F_{\varphi}\left(X_{n} g\right)-F_{\varphi}\left(X_{n}\right)\right|^{2} d \mu^{* r}(g) d \mathbf{P}(\omega)=\mu^{* n+r}\left(F_{\varphi}^{2}\right)-\mu^{* n}\left(F_{\varphi}^{2}\right)
$$

because $\int F_{\varphi}\left(X_{n} g\right) d \mu^{* r}(g)=F_{\varphi}\left(X_{n}\right)$. One deduces that

$$
\iint \sum_{n=0}^{p}\left|F_{\varphi}\left(X_{n} g\right)-F_{\varphi}\left(X_{n}\right)\right|^{2} d \mathbf{P}(\omega) d \mu^{* r}(g) \leq 2 r\|\varphi\|_{\infty}^{2}
$$

for every $r \geq 1$; this proves the convergence $\mathbf{P} \otimes \mu^{* r}$-a.e. of the series $\sum_{n=0}^{\infty}\left|F_{\varphi}\left(X_{n} g\right)-F_{\varphi}\left(X_{n}\right)\right|^{2}$ and consequently the convergence of $F_{\varphi}\left(X_{n} g\right)-$ $F_{\varphi}\left(X_{n}\right)$ to zero.

In what follows we are going to use concepts introduced in [13]. Therefore, we recall them briefly.

To every linear transformation of $\mathbb{R}^{d}$ is associated a quasi-projective transformation acting on the lines of $\mathbb{R}^{d}$ not contained in the kernel of the transformation. So we have maps of $\mathbb{P}^{d-1}$ defined outside a projective subspace: these maps are continuous outside the exceptional subspace and are limits, outside this subspace, of a sequence of projective transformations. Furthermore, from every sequence of projective transformations, we can extract a subsequence converging to a quasi-projective one, outside a projective subspace.

TheOREM 5.4. Let $\nu$ be a $\mu$-stationary measure on $\mathbb{P}(V)$. Assume that $\Gamma=\Gamma_{\mu}$ satisfies conditions $\left(H_{1}\right)$ and $\left(H_{2}\right)$. Then we have $\mathbf{P}_{\mu}$-a.e.

$$
\lim _{n} g_{1} g_{2} \ldots g_{n} . \nu=\delta_{z_{\omega}} .
$$

In particular $\nu$ is unique and its support is $L_{\Gamma}$.

Proof. The proof goes as in [17]. For a fixed $\omega$, we consider the relation given by Proposition 5.3,

$$
\theta(\omega)=\lim _{n} g_{1}(\omega) \ldots g_{n}(\omega) . \nu=\lim _{n} g_{1}(\omega) \ldots g_{n}(\omega) g . \nu,
$$


which is true for $\mathbf{P}_{\mu} \otimes \eta$-a.e. $(\omega, g)$. One can extract from $g_{1}(\omega) \ldots g_{n}(\omega)$ a subsequence converging outside a projective subspace to a quasi-projective map $\tau(\omega)$. As $\nu$ gives zero measure to any projective subspace (Lemma 5.1), from Proposition 5.3 above one has $\tau(\omega) . \nu=\tau(\omega) \gamma . \nu=\theta(\omega)$ for $\eta$-a.e. $\gamma$, and therefore for all $\gamma \in \Gamma$. As $\Gamma$ satisfies $\left(H_{1}\right)$ and $\left(H_{2}\right)$, one can find a sequence $\gamma_{n} \in \Gamma$ such that $\gamma_{n} . \nu$ converges to a Dirac measure $\delta_{z}$ with $z$ belonging to the open set of continuity of $\tau(\omega)$. Then, in the limit $\theta(\omega)=\tau(\omega) . \delta_{z}$. This proves that $\theta(\omega)$ is a Dirac measure $\delta_{z_{\omega}}$. The law of the random variable $z$ is necessarily $\nu$ by the martingale convergence theorem. Since $z$ is independent of the choice of the $\mu$-stationary measure $\nu$ we get the uniqueness of $\nu$.

Clearly, $S_{\nu}$ is closed and $\Gamma$-invariant. Hence, Proposition 4.5 shows that $S_{\nu} \supset L_{\Gamma}$. The Markov-Kakutani theorem and uniqueness of $\nu$ give, as in the proof of Lemma 5.1, $\nu\left(L_{\Gamma}\right)=1$, hence $S_{\nu}=L_{\Gamma}$.

Corollary 5.5. Let $\varrho\left(\varrho^{*}\right.$ resp. $)$ be a probability measure on $\mathbb{P}(V)\left(\mathbb{P}\left(V^{*}\right)\right.$ resp.) which gives zero mass to every projective subspace. Then we have $\mathbf{P}_{\mu^{-}}$ a.e.

$$
\lim _{n} g_{1} \ldots g_{n} \cdot \varrho=\delta_{z_{\omega}} \quad\left(\lim _{n} g_{1}^{t} \ldots g_{n}^{t} \cdot \varrho^{*}=\delta_{z_{\omega}^{*}} \text { resp. }\right) .
$$

In particular

$$
\lim _{n} g_{1} \ldots g_{n} . m=\delta_{z_{\omega}} \quad\left(\lim _{n} g_{1}^{t} \ldots g_{n}^{t} \cdot m^{*}=\delta_{z_{\omega}^{*}} \text { resp. }\right),
$$

where $m\left(m^{*}\right.$ resp. $)$ is the $K$-invariant probability measure on $\mathbb{P}(V)\left(\mathbb{P}\left(V^{*}\right)\right.$ resp.)

Proof. We observe that $g_{1} \ldots g_{n} /\left\|g_{1} \ldots g_{n}\right\| \in \operatorname{End}(V)$ has norm one, and consider an arbitrary convergent subsequence,

$$
u=\lim _{k} \frac{g_{1} \ldots g_{n_{k}}}{\left\|g_{1} \ldots g_{n_{k}}\right\|} .
$$

Clearly $u \neq 0$, since $\|u\|=1$. We note that $u$ defines a continuous map from $\mathbb{P}(V) \backslash \operatorname{Ker} u$ into $\mathbb{P}(V)$. We will denote it again by $u$, and observe that, since $\varrho(\operatorname{Ker} u)=0, u . \varrho$ is well defined, and from dominated convergence,

$$
u . \varrho=\lim _{k} g_{1} \ldots g_{n_{k}} \cdot \varrho .
$$

In particular, from Theorem 5.4 and Lemma 5.1, u. $\nu=\delta_{z_{\omega}}$. This means that the linear map $u$ has rank one and satisfies $u(\mathbb{P}(V) \backslash \operatorname{Ker} u)=\delta_{z_{\omega}}$. Hence $u . \varrho=\delta_{z_{\omega}}$. The convergent subsequence chosen above was arbitrary, hence

$$
\lim _{n} g_{1} \ldots g_{n} \cdot \varrho=\delta_{z_{\omega}} \text {. }
$$

In particular, we have the above convergence for $\varrho=m$.

The results for $\mathbb{P}\left(V^{*}\right), \varrho^{*}, m^{*}, z_{\omega}^{*}$ follow from $\Gamma_{\mu^{*}}=\left(\Gamma_{\mu}\right)^{t}$ and Remark 2.1(iii). 
Recall $m$ is the $K$-invariant probability measure on $\mathbb{P}(V)$, where $K=$ $\mathrm{SO}(d, \mathbb{R})$. One says that a sequence $f_{n} \in \mathrm{GL}(d, \mathbb{R})$ has the contraction property on $\mathbb{P}(V)$ towards $z$ if the sequence of measures $f_{n} . m$ on $\mathbb{P}(V)$ converges weakly towards $\delta_{z}$. A point $z \in \mathbb{P}(V)$ will be identified with a vector of norm one, defined up to sign.

We will use, as in [17] and [18], the $K \overline{A^{+}} K$ decomposition of $g \in \mathrm{GL}(d, \mathbb{R})$, $g=k a k^{\prime}, k$ and $k^{\prime}$ are orthogonal matrices and $\overline{A^{+}} \ni a=\operatorname{diag}\left(a^{1}, \ldots, a^{d}\right)$ with $a^{1} \geq \cdots \geq a^{d}$. Let $\left(e_{1}, \ldots, e_{d}\right)$ denote the canonical basis of $\mathbb{R}^{d}$. In particular, if $g \in \mathrm{SL}(d, \mathbb{R})$ then $k, k^{\prime} \in K=\mathrm{SO}(d, \mathbb{R})$ and $a \in \overline{A^{+}}=$ $\left\{\operatorname{diag}\left(a^{1}, \ldots, a^{d}\right): a^{1} \geq \cdots \geq a^{d}>0\right.$ and $\left.\prod_{i=1}^{d} a^{i}=1\right\}$.

If one writes the polar decomposition of $f_{n}$ as $f_{n}=k_{n} a_{n} k_{n}^{\prime}$, where $k_{n}, k_{n}^{\prime} \in K, a \in \overline{A^{+}}$, one sees that the contraction property is equivalent to $a_{n}^{i}=o\left(a_{n}^{1}\right), 1<i \leq d, \lim _{n} k_{n} \cdot e_{1}=z$.

In the proposition below and its corollary, the point $z \in \mathbb{P}\left(V^{*}\right)$ is considered as a unit vector, hence $|z(x)|$ is well defined for $x \in V$.

Proposition 5.6. Assume that $f_{n} \in \mathrm{GL}(V)$ is a sequence such that $f_{n}^{t}$ has the contraction property on $\mathbb{P}\left(V^{*}\right)$ towards $z \in \mathbb{P}\left(V^{*}\right)$. Then for any $x, y \in \mathbb{P}(V)$,

$$
\lim _{n} \frac{\left\|f_{n}(x)\right\|}{\left\|f_{n}\right\|}=|z(x)|, \quad \lim _{n} z(x) z(y) \frac{\delta\left(f_{n} \cdot x, f_{n} \cdot y\right)}{\delta(x, y)}=0 .
$$

The second convergence is uniform when $x, y$ belong to a compact subset of $\mathbb{P}(V) \backslash \operatorname{Ker} z$. If $f_{n} \in \mathrm{SL}(V)$, then $\lim _{n}\left\|f_{n}(x)\right\|=+\infty$ for every $x \notin \operatorname{Ker} z$.

Proof. Recall that the distance between $\bar{u}=\pi(u)$ and $\bar{v}=\pi(v)$ in $\mathbb{P}(V)$ is equal to $\delta(\bar{u}, \bar{v})=\|u \wedge v\| /\|u\|\|v\|$.

One writes $f_{n}=k_{n} a_{n} k_{n}^{\prime}$ as above, with $k_{n}, k_{n}^{\prime} \in K$ and $a_{n} \in \overline{A^{+}}$. From the contraction property of $f_{n}^{t}$ we get

$$
\lim _{n} k_{n}^{\prime-1} \cdot \bar{e}_{1}=z, \quad a_{n}^{i}=o\left(a_{n}^{1}\right) \quad(i>1) .
$$

Writing $x=\sum_{i=1}^{d} x_{i} e_{i}$, we get

$$
\left\|f_{n} x\right\|^{2}=\left\|a_{n} k_{n}^{\prime} x\right\|^{2}=\sum_{i=1}^{d}\left(a_{n}^{i}\right)^{2}\left|\left\langle k_{n}^{\prime} x, e_{i}\right\rangle\right|^{2} \geq\left(a_{n}^{1}\right)^{2}\left|\left\langle k_{n}^{\prime} x, e_{1}\right\rangle\right|^{2} .
$$

Since the norm of $f_{n}$ is $a_{n}^{1}$, we get

$$
\begin{aligned}
\lim _{n} \frac{\left\|f_{n} x\right\|^{2}}{\left\|f_{n}\right\|^{2}} & =\lim _{n}\left|\left\langle x, k_{n}^{\prime-1} e_{1}\right\rangle\right|^{2}+\lim _{n} \sum_{i>1}\left(\frac{a_{n}^{i}}{a_{n}^{1}}\right)^{2}\left|\left\langle k_{n}^{\prime} x, e_{1}\right\rangle\right|^{2} \\
& =\lim _{n}\left|\left\langle x, k_{n}^{\prime-1} e_{1}\right\rangle\right|^{2}=|z(x)|^{2} .
\end{aligned}
$$


Also

$$
\begin{aligned}
&\left\|f_{n}(x) \wedge f_{n}(y)\right\|^{2}=\sum_{i \neq j}\left(a_{m}^{i} a_{n}^{j}\right)^{2}\left|\left\langle k_{n}^{\prime}(x \wedge y), e_{i} \wedge e_{j}\right\rangle\right|^{2}, \\
&\left\|f_{n}(x) \wedge f_{n}(y)\right\| \leq d^{2} a_{n}^{1} a_{n}^{2}\|x \wedge y\| .
\end{aligned}
$$

Therefore,

$$
\frac{\delta\left(f_{n} \cdot x, f_{n} \cdot y\right)}{\delta(x, y)}=\frac{\left\|f_{n} x \wedge f_{n} y\right\|}{\left\|f_{n} x\right\|\left\|f_{n} y\right\|\|x \wedge y\|} \leq d^{2} \frac{a_{n}^{2}}{a_{n}^{1}} \frac{1}{\left|\left\langle k_{n}^{\prime} x, e_{1}\right\rangle\left\langle k_{n}^{\prime} y, e_{1}\right\rangle\right|}
$$

and

$$
\lim _{n}|z(x)||z(y)| \frac{\delta\left(f_{n} \cdot x, f_{n} \cdot y\right)}{\delta(x, y)}=0 .
$$

The uniformity of the required convergence is clear from the previous formula if $z(x) z(y) \not \equiv 0$.

In order to obtain the last assertion, it suffices to show, in view of the first statement, that $\left\|f_{n}\right\|$ converges to $\infty$. The relation $a_{n}^{2}=o\left(a_{n}^{1}\right)$ implies $\operatorname{det} f_{n}=\prod_{i=1}^{d} a_{n}^{i}=o\left(a_{n}^{1}\right)$. Since $\operatorname{det} f_{n}=1$, we conclude that $\lim _{n}\left\|f_{n}\right\|=$ $\lim _{n} a_{n}^{1}=\infty$.

Now we are able to get information about the vector $S_{n}(\omega) x$, if $x$ is fixed, as follows:

Corollary 5.7 (see $[17,18]$ ). If $\mu, z_{\omega}^{*}$ are as in Theorem 5.4 and Corollary 5.5, then, as $n$ tends to infinity, we have uniformly in $x, y \in \mathbb{P}(V)$,

$$
\lim _{n} \mathbf{E}_{\mu} \delta\left(S_{n} \cdot x, S_{n} \cdot y\right)=0,
$$

and $\mathbf{P}_{\mu}$-a.e.

$$
\lim _{n} \frac{\left\|S_{n} x\right\|}{\left\|S_{n}\right\|}=\left|z_{\omega}^{*}(x)\right| .
$$

If $\mu \in M^{1}(\mathrm{SL}(V))$, then, for every $x \in V$ and $\mathbf{P}_{\mu^{-}}$a.e., $\lim _{n}\left\|S_{n} x\right\|=\infty$.

Proof. Note that Corollary 5.5 implies that $S_{n}^{t}(\omega)$ has the contraction property towards $z_{\omega}^{*}$. Hence Proposition 5.6 implies

$$
\lim _{n} \frac{\left\|S_{n} x\right\|}{\left\|S_{n}\right\|}=\left|z_{\omega}^{*}(x)\right| .
$$

For the first convergence it suffices to show that for any sequence $x_{n}, y_{n} \in$ $\mathbb{P}(V)$ we have $\mathbf{P}_{\mu}$-a.e.

$$
\lim _{n} \delta\left(S_{n} \cdot x_{n}, S_{n} \cdot y_{n}\right)=0
$$

Then the first formula will follow from dominated convergence.

One can suppose that $\lim _{n} x_{n}=x$ and $\lim _{n} y_{n}=y$. From Corollary 5.5 and Lemma 5.1, one knows that the law of $z_{\omega}^{*}=\lim _{n} S_{n}^{t} \cdot m^{*}$ gives zero measure to every subspace. Hence, for almost every $\omega \in \Omega, x$ and $y$ are not 
in Ker $z_{\omega}^{*}$, and the same is true for $x_{n}, y_{n}$ for large $n$. Then (5.8) follows from the second formula in Proposition 5.6.

The last assertion is proved as follows. If $x$ is fixed, then $\mathbf{P}_{\mu}$-a.e. as above $\left|z_{\omega}^{*}(x)\right| \neq 0$, hence from the first formula in Proposition 5.6 it follows that $\lim _{n}\left\|S_{n} x\right\|=\infty$.

REMARK 5.9. In [18] (see Theorem 4.4) the first and the last conclusion of Corollary 5.7 is proved under a weaker hypothesis, namely validity of $\left(\mathrm{H}_{2}\right)$ is replaced by non-relative compactness of $\Gamma=\Gamma_{\mu}$. Such a result can also be deduced from Corollary 5.7, using wedge products.

Now we are going to study stationary measures on factor spaces of $V \backslash\{0\}$.

Fix $c>1$ and denote by $\mathbb{P}_{c}(V)=\mathbb{P}_{c}^{d-1}$ the factor space of $V \backslash\{0\}$ by the multiplicative subgroup

$$
\pm c^{\mathbb{Z}}:=\left\{ \pm c^{n}: n \in \mathbb{Z}\right\}
$$

of $\mathbb{R}^{*}$ and denote by $\mathbb{T}_{c}$ the 1 -torus $\mathbb{R}^{*} / \pm c^{\mathbb{Z}}$.

We can consider the projection from $V \backslash\{0\}$ to $\mathbb{P}(V) \times \mathbb{T}_{c}$ given by

$$
v \mapsto\left(\bar{v},\|v\|^{i \alpha}\right),
$$

where $\alpha=2 \pi / \log c$ and we observe that $\mathbb{P}_{c}(V)$ is then naturally identified with $\mathbb{P}(V) \times \mathbb{T}_{c}$. Hence a point of $\mathbb{P}_{c}(V)$ will be written as $v=(\bar{v}, z)$, where $\bar{v} \in \mathbb{P}(V)$ is the projection of $v$ and $z=\|v\|^{i \alpha}$. The action of $g \in G=\operatorname{GL}(V)$ on $\mathbb{P}_{c}(V)$ can then be written as

$$
g \cdot v=g \cdot(\bar{v}, z)=\left(g \cdot \bar{v}, z\|g \bar{v}\|^{i \alpha}\right) .
$$

$\mathbb{R}^{*}$ acts also on this space and the two actions commute. The corresponding formula is

$$
t .(\bar{v}, z)=\left(\bar{v}, z|t|^{i \alpha}\right), \quad t \in \mathbb{R}^{*} .
$$

We denote by $\lambda_{c}=d z$ the normalized Lebesgue measure on $\mathbb{T}_{c}$ and observe that every measure of the form $\nu \otimes \lambda_{c}$, where $\nu \in M^{1}(\mathbb{P}(V))$, is invariant under the action of $\mathbb{R}^{*}$ on $\mathbb{P}_{c}(V)$. Furthermore, if $\mu \in M^{1}(G)$ and $\nu \in M^{1}(\mathbb{P}(V))$ is $\mu$-stationary, then $\nu \otimes \lambda_{c}$ is also $\mu$-stationary. If $L_{\Gamma} \subset \mathbb{P}(V)$ is the asymptotic set of $\Gamma\left(\Gamma=\Gamma_{\mu}\right.$ satisfies conditions $\left(H_{1}\right)$ and $\left.\left(H_{2}\right)\right)$, then $L_{\Gamma}(c)=L_{\Gamma} \times \mathbb{T}_{c}$ is a closed and $\Gamma$-invariant subset of $\mathbb{P}(V) \times \mathbb{T}_{c}$.

Theorem 5.10. Assume that $\mu \in M^{1}(G)$ is such that $\Gamma=\Gamma_{\mu}$ satisfies conditions $\left(H_{1}\right)$ and $\left(H_{2}\right)$. Then, with the above notations, for every $\psi \in$ $C\left(\mathbb{P}_{c}(V)\right)$ the sequence $\check{\mu}^{* n} * \psi$ converges uniformly to $\left(\nu \otimes \lambda_{c}\right)(\psi)$, where $\nu$ is the $\mu$-stationary measure on $\mathbb{P}(V)$. Furthermore, for any $v \in \mathbb{P}_{c}(V)$ we have the following a.e. convergence:

$$
\lim _{n} \delta^{c}\left(S_{n}(\omega) \cdot v, L_{\Gamma}(c)\right)=0,
$$


where $\delta^{c}$ is the distance on $\mathbb{P}(V) \times \mathbb{T}_{c}$ given by

$$
\delta^{c}\left(v, v^{\prime}\right)=\delta\left(\bar{v}, \overline{v^{\prime}}\right)+\left|z-z^{\prime}\right|,
$$

and $v=(\bar{v}, z), v^{\prime}=\left(\overline{v^{\prime}}, z^{\prime}\right)$.

Corollary 5.12. Assume $\Gamma \subset G$ is a subsemigroup of $G$ which satisfies conditions $\left(H_{1}\right)$ and $\left(H_{2}\right)$ and $c>1$ is fixed. Then the closed $\Gamma$-invariant subset $L_{\Gamma}(c)=L_{\Gamma} \times \mathbb{T}_{c}$ of $\mathbb{P}_{c}(V)$ is the unique minimal set. Furthermore, any $\mu \in M^{1}(G)$ such that $\Gamma=\Gamma_{\mu}$ satisfies conditions $\left(H_{1}\right)$ and $\left(H_{2}\right)$ has a unique stationary measure on $\mathbb{P}_{c}(V)$.

Clearly, Theorem 5.10 and its corollary imply Theorem 1.3 of the Introduction.

For the proof of Theorem 5.10 we need three lemmas.

LEMMA 5.13. If $\mu$ is as in Theorem 5.10 then for $x, y \in V$,

$$
\lim _{y \rightarrow x} \limsup _{n} \mathbf{E}_{\mu}\left|\left(\frac{\left\|S_{n} x\right\|}{\left\|S_{n} y\right\|}\right)^{i \alpha}-1\right|=0 .
$$

Proof. From Corollary 5.7 we know that if $x_{n} \rightarrow x$ and $y_{n} \rightarrow y$, then

$$
\lim _{n} \frac{\left\|S_{n} x_{n}\right\|}{\left\|S_{n} y_{n}\right\|}=\frac{\left|z_{\omega}(x)\right|}{\left|z_{\omega}(y)\right|}
$$

Hence, from dominated convergence,

$$
\underset{n}{\limsup } \mathbf{E}_{\mu}\left|\left(\frac{\left\|S_{n} x_{n}\right\|}{\left\|S_{n} y_{n}\right\|}\right)^{i \alpha}-1\right|=\left.\mathbf{E}_{\mu}|| \frac{z_{\omega}(x)}{z_{\omega}(y)}\right|^{i \alpha}-1 \mid .
$$

The formula in the lemma corresponds to the special case $x_{n}=x, y=x$.

LEMMA 5.14. If $\mu$ is as in Theorem 5.10, then for every $\psi \in C\left(\mathbb{P}_{c}(V)\right)$ the sequence of functions $\check{\mu}^{* k} * \psi$ is uniformly equicontinuous.

Proof. One considers the distance $\delta^{c}$ on $\mathbb{P}_{c}(V)$ given by (5.11). Then, in view of the form of the action of $G$ on $\mathbb{P}_{c}(V)$,

$$
\delta^{c}\left(S_{n} \cdot v, S_{n} \cdot v^{\prime}\right)=\delta\left(S_{n} \cdot \bar{v}, S_{n} \cdot \overline{v^{\prime}}\right)+\left|\left(\frac{\left\|S_{n} \bar{v}\right\|}{\left\|S_{n} \overline{v^{\prime}}\right\|}\right)^{i \alpha}-1\right|+\left|z-z^{\prime}\right| .
$$

From the proof of Lemma 5.13, we get

$$
\lim _{n} \delta^{c}\left(S_{n} \cdot v, S_{n} \cdot v^{\prime}\right)=\left.|| \frac{\left\langle\bar{v}, z_{\omega}^{*}\right\rangle}{\left\langle\overline{v^{\prime}}, z_{\omega}^{*}\right\rangle}\right|^{i \alpha}-1|+| z-z^{\prime} \mid .
$$

Hence, by dominated convergence,

$$
\limsup _{n} \mathbf{E}_{\mu} \delta^{c}\left(S_{n} \cdot v, S_{n} \cdot v^{\prime}\right)=\left|z-z^{\prime}\right|+\mathbf{E}_{\mu}\left(\left|\frac{\left\langle\bar{v}, z_{\omega}^{*}\right\rangle}{\left\langle\overline{v^{\prime}}, z_{\omega}^{*}\right\rangle}\right|^{i \alpha}-1\right) .
$$

The right hand side of this formula is uniformly small when $\delta^{c}\left(v, v^{\prime}\right)$ is small.

Now, if $\psi \in C\left(\mathbb{P}_{c}(V)\right)$ is Lipschitz, with coefficient $[\psi]$, then

$$
\left|\check{\mu}^{* n} * \psi(v)-\check{\mu}^{* n} * \psi\left(v^{\prime}\right)\right| \leq \mathbf{E}_{\mu}\left[\delta^{c}\left(S_{n} \cdot v, S_{n} \cdot v^{\prime}\right)\right][\psi] .
$$


Since Lipschitz functions are dense in $C\left(\mathbb{P}_{c}(V)\right)$ the above inequality and (5.15) imply equicontinuity of the sequence $\check{\mu}^{* n} * \psi(v)$ for $\psi \in C\left(\mathbb{P}_{c}(V)\right)$.

Lemma 5.16. Suppose $\theta \in \mathbb{R}, \eta \in C(\mathbb{P}(V))$ and $\eta \not \equiv 0$ and satisfies the equation

$$
\int \eta(g \cdot \bar{v})\|g \bar{v}\|^{i \alpha} d \mu(g)=e^{i \theta} \eta(\bar{v}) .
$$

Then $\alpha=0, \theta=0$ and $\eta=$ const on $\mathbb{P}(V)$.

Proof. Passing to absolute values in (5.17) we get

$$
|\eta(\bar{v})| \leq \int|\eta(g \cdot \bar{v})| d \mu(g) .
$$

Let $M=\left\{\bar{v} \in \mathbb{P}(V):|\eta(\bar{v})|=\|\eta\|_{\infty}\right\}$. Then from (5.18) the condition $\bar{v} \in M$ implies $g . \bar{v} \in M \mu$-a.e. Hence from continuity of $|\eta|$, we have $g . M \subset M$ for every $g \in S_{\mu}$ and $\Gamma_{\mu} . M \subset M$. Since $L_{\Gamma}$ is the unique minimal subset in $\mathbb{P}(V)$ (see Proposition 4.5), we get $L_{\Gamma} \subset M$. In particular, $|\eta(\bar{v})|=\|\eta\|_{\infty}$ for every $\bar{v} \in L_{\Gamma}$. From strong convexity of the unit disc in $\mathbb{C}$ and (5.17) we get

$$
\forall \bar{v} \in L_{\Gamma}, \forall g \in S_{\mu}, \quad \eta(g \cdot \bar{v})\|g \bar{v}\|^{i \alpha}=e^{i \theta} \eta(\bar{v}) .
$$

From Proposition 4.6 it follows that $\alpha=0, \theta=0$ and $\eta=$ const on $L_{\Gamma}$.

Now on $\mathbb{P}(V)$ we have

$$
\int \eta(g \cdot \bar{v}) d \mu(g)=\eta(\bar{v}) .
$$

We can suppose $\eta$ to be real and we consider the set $M^{\prime}\left(M^{\prime \prime}\right.$ resp.) of points where $\eta$ attains its maximum (minimum resp.). As above we obtain $M^{\prime} \supset L_{\Gamma}$. Replacing $\eta$ by $-\eta$, we also obtain $M^{\prime \prime} \supset L_{\Gamma}$, hence $M^{\prime}=M^{\prime \prime}$. We conclude that

$$
\forall \bar{v} \in \mathbb{P}(V), \quad \eta(\bar{v})=\text { const. }
$$

Proof of Theorem 5.10. We use the following result of [28]. Let $P$ be a Markov operator on the compact metric space $X$, which preserves $C(X)$ and is equicontinuous, i.e., for any $\psi \in C(X)$, the sequence $P^{k} \psi, k \in \mathbb{N}$, is equicontinuous. Then if 1 is the only eigenvalue of modulus one in $C(X)$, the sequence $P^{k} \psi$ converges uniformly. Here we have $P(x, \cdot)=\mu * \delta_{x}$, and $X=\mathbb{P}_{c}(V)$. From Lemma 5.14 we know that $P$ is equicontinuous. Suppose that $\eta \in C(X)$ with $\eta \not \equiv 0$ satisfies $P \eta=e^{i \theta} \eta$, i.e.,

$$
\int \eta(g . v) d \mu(g)=e^{i \theta} \eta(v)
$$

for any $v$ in $\mathbb{P}_{c}(V)$. Now we can consider the Fourier coefficients $(k \in \mathbb{Z})$

$$
\eta_{k}(\bar{v})=\int \eta(\bar{v}, z) z^{k} d \lambda_{c}(z)
$$

and we obtain

$$
\int \eta_{k}(g \cdot \bar{v})\|g \bar{v}\|^{i k \alpha} d \mu(g)=e^{i \theta} \eta_{k}(\bar{v})
$$


From Lemma 5.16 we get $e^{i \theta}=1, \eta_{k}(\bar{v})=0$ for $k \neq 0, \eta_{0}(\bar{v}) \equiv$ const. Hence $\eta=$ const on $\mathbb{P}_{c}(V)$. Now the result of [28], recalled above, gives the uniform convergence of the sequence $\psi_{n}=\check{\mu}^{* n} * \psi$.

Clearly, if $\lim _{n} \psi_{n}=\eta$, one has $P \eta=\check{\mu} * \eta=\eta$ and $\eta$ is continuous. From the above result, we deduce $\eta \equiv$ const. Furthermore,

$$
\eta=\left(\nu \otimes \lambda_{c}\right)(\eta)=\lim _{n}\left(\nu \otimes \lambda_{c}\right)\left(\psi_{n}\right)=\left(\nu \otimes \lambda_{c}\right)(\psi) .
$$

Hence we obtain the formula $\eta=\left(\nu \otimes \lambda_{c}\right)(\psi)$ and the required convergence.

In order to prove the second statement of the theorem, notice that since $L_{\Gamma}(c)$ is the inverse image of $L_{\Gamma}$ in $\mathbb{P}_{c}(V)$ we have

$$
\delta^{c}\left(S_{n}(\omega) \cdot v, L_{\Gamma}(c)\right)=\delta\left(S_{n}(\omega) \cdot \bar{v}, L_{\Gamma}\right) .
$$

Proposition 5.6 implies that, given $\bar{v}$ and $\bar{w}$ in $\mathbb{P}(V)$, we have the a.e. convergence of the sequence $\delta\left(S_{n}(\omega) \cdot \bar{v}, S_{n}(\omega) \cdot \bar{w}\right)$ to zero. If we choose $\bar{w}$ in $L_{\Gamma}$, then $S_{n}(\omega) \cdot \bar{w} \in L_{\Gamma}$, hence

$$
\delta\left(S_{n}(\omega) \cdot \bar{v}, L_{\Gamma}\right) \leq \delta\left(S_{n}(\omega) \cdot \bar{v}, S_{n}(\omega) \cdot \bar{w}\right) .
$$

It follows that $\lim _{n} \delta\left(S_{n}(\omega) \cdot \bar{v}, L_{\Gamma}\right)=\delta\left(S_{n}(\omega) \cdot \bar{v}, S_{n}(\omega) \cdot \bar{w}\right)=0$.

Proof of Corollary 5.12. Suppose $\xi \in M^{1}\left(\mathbb{P}_{c}(V)\right)$ is another $\mu$-stationary measure. Since $\psi_{n}=\check{\mu}^{* n} * \psi$ converges uniformly to $\left(\nu \otimes \lambda_{c}\right)(\psi)$, we get

$$
\xi\left(\lim _{n} \psi_{n}\right)=\lim _{n} \xi\left(\check{\mu}^{* n} * \psi\right)=\xi(\psi) .
$$

Hence, $\left(\nu \otimes \lambda_{c}\right)(\psi)=\xi(\psi), \nu \otimes \lambda_{c}=\xi$ and the uniqueness follows.

Suppose $\Delta$ is a closed $\Gamma_{\mu}$-invariant subset of $\mathbb{P}_{c}(V)$. Then from the Markov-Kakutani theorem, there is a $\mu$-stationary measure carried by $\Delta$. From the uniqueness of the stationary measure we get

$$
\Delta \supset \operatorname{supp} \nu \otimes \lambda_{c}=L_{\Gamma}(c) .
$$

Hence $L_{\Gamma}(c)$ is the unique $\Gamma$-minimal subset of $\mathbb{P}_{c}(V)$.

ThEOREM 5.19. Suppose that $\Gamma$ is a subsemigroup of $\mathrm{GL}(d, \mathbb{R}), d>1$, satisfying conditions $\left(H_{0}\right),\left(H_{1}\right)$ and $\left(H_{2}\right)$, and let $\Sigma$ be $\Gamma$-invariant subset of $\widetilde{V} \backslash\{0\}$ such that 0 is a limit point of $\Sigma$. Then

$$
\bar{\Sigma} \supset \widetilde{L}_{\Gamma} /\{\operatorname{Id}, \sigma\} \text {. }
$$

Proof. We denote by $\Sigma^{\prime}$ the inverse image of $\Sigma$ in $V \backslash\{0\}$. Let $u_{0}$ be a $\gamma$-dominant vector as in Proposition 4.1, that is, satisfying

$$
\gamma^{\mathbb{Z}} u_{0}:=\left\{\gamma^{k} u_{0}: k \in \mathbb{Z}\right\} \subset \overline{\Sigma^{\prime}} .
$$

Applying Corollary 5.12 with $c=\lambda$, where $\lambda$ is the unique eigenvalue of $\gamma$ of maximum modulus, greater than 1 since $\gamma$ is quasi-expanding, we find that if $\bar{u}_{0}$ denotes the projection of $u_{0}$ on $\mathbb{P}_{c}(V)$ then $\overline{\Gamma \bar{u}_{0}} \supset L_{\Gamma}(c)$. It follows that if $\bar{y} \in L_{\Gamma}(c)$ is given, then there is a sequence $\left\{\gamma_{n}\right\} \subset \Gamma$ such that $\gamma_{n} . \overline{u_{0}}$ 
converges to $\bar{y}$. This implies that there is a sequence $\left\{p_{n}\right\}$ of integers such that $\lambda^{p_{n}} \gamma_{n} u_{0} \rightarrow y \in V \backslash\{0\}$, which implies

$$
\gamma_{n} \lambda^{p_{n}} u_{0}=\gamma_{n} \gamma^{p_{n}} u_{0} \rightarrow y .
$$

But $\gamma^{p_{n}} u_{0} \in \overline{\Sigma^{\prime}}$ by (5.21). Thus $y \in \overline{\Sigma^{\prime}}$. Since $\bar{y}$ was an arbitrary point from $L_{\Gamma}(c)$ we conclude that $\widetilde{L}_{\Gamma} \subset \overline{\Sigma^{\prime}}$ and $(5.20)$ is proved.

Clearly, Theorem 5.19 gives Theorem 1.2 of the Introduction.

Theorem 5.19 will be used below in the following special case. In view of Remark 2.1(ii) condition $\left(H_{0}\right)$ is satisfied in this case.

Corollary 5.22. Let $\Gamma$ be a subsemigroup of $\mathrm{M}_{\mathrm{inv}}(d, \mathbb{Z}), d>1$, satisfying $\left(H_{1}\right)$ and $\left(H_{2}\right)$. Let $\Sigma$ be a $\Gamma$-invariant subset of $\widetilde{V} \backslash\{0\}$ such that 0 is a limit point of $\Sigma$. Then $\bar{\Sigma} \supset \widetilde{L}_{\Gamma} /\{\mathrm{Id}, \sigma\}$.

Theorem 5.19 does not give information on a general $\Gamma$-orbit closure $\overline{\Gamma v}, v \in \widetilde{V} \backslash\{0\}$, if 0 is not a limit point. On the other hand, Theorem 5.10 and its corollary describe the behavior of a general $\Gamma$-orbit in $\mathbb{P}_{c}(V)$. Using more precise information on products of random matrices, i.e. the renewal theorem as in [19] (see also [22]), one can go further and describe the behavior at infinity of a general orbit $\Gamma v \subset \widetilde{V} \backslash\{0\}$ as follows. For any $c, d(1 \leq c<d)$ we denote by $\widetilde{V}_{[c, d]} \subset \widetilde{V} \backslash\{0\}$ the " $c$-shell" $\mathbb{P}^{d-1} \times[c, d]$, and by $\widetilde{L}_{\Gamma, c} \subset \widetilde{V}_{c}:=\mathbb{P}^{d-1} \times[1, c]$ the closed subset $L_{\Gamma} \times[1, c]$. Then by the methods of [7] and [19] we can obtain the following

TheOREM 5.23. Assume that the semigroup $\Gamma \subset \mathrm{GL}(d, \mathbb{R}), d>1$, satisfies $\left(H_{0}\right),\left(H_{1}\right)$ and $\left(H_{2}\right)$. With the above notations, for any $c>1$ and $v \in \widetilde{V} \backslash\{0\}$ every cluster value of the family of closed sets $c^{-t}\left(\overline{\Gamma v} \cap \widetilde{V}_{\left[c^{t}, c^{t+1}\right]}\right)$ contains $\widetilde{L}_{\Gamma, c}$.

This can be interpreted as "thickness" at infinity, in the direction of $\widetilde{L}_{\Gamma}$, of the orbit closure $\overline{\Gamma v} \subset \widetilde{V}$.

Theorems 5.19 and Corollary 5.22 can also be deduced from Theorem 5.23 .

REMARK 5.24. The conclusions in statements 5.19 to 5.23 are also valid if $d=1$, if one supposes the semigroup $\Gamma$ of $\mathbb{R}^{*}$ to be non-lacunary. The corresponding aperiodicity condition in the statements above is automatically satisfied if $d>1$, because of Corollary 4.8.

6. Proof of Theorem 1.1. In order to prove the theorem, we use ideas of [12] and [4]. The first step is to prove that if $\Sigma \subset \mathbb{T}^{d}$ is a closed $\Gamma$-invariant subset that contains $0 \in \mathbb{T}^{d}$ as a limit point, then $\Sigma=\mathbb{T}^{d}$. Here we suppose $\Gamma \subset \mathrm{M}_{\text {inv }}(d, \mathbb{Z})$ and we apply Corollary 5.22 to the inverse image $p^{-1}(\Sigma)$ of $\Sigma$ in $\mathbb{R}^{d}$. 
In the general case, we suppose $\Sigma$ to be infinite and we construct other closely related closed $\Gamma$-invariant subsets of $\mathbb{T}^{d}$ which contain 0 . Then we use the special case above to get information on $\Sigma$ and we conclude that $\Sigma=\mathbb{T}^{d}$.

6.1. The case when 0 is a limit point of $\Sigma$. The statement $\Sigma=\mathbb{T}^{d}$ will hold by Corollary 5.22 applied to $p^{-1}(\Sigma)$ if we are able to see that $\widetilde{L}_{\Gamma}$ contains at least one ray which is not contained in a rational subspace. But the set of rational subspaces is countable and, by Lemma 5.1, $L_{\Gamma}$ is not contained in a countable union of subspaces. The result follows.

We can observe that the set $\widetilde{L}_{\Gamma}$ is very large, since it was proved in [6] that $L_{\Gamma}$ has strictly positive Hausdorff dimension.

6.2. The general case. In order to show that the special case above is the only one, we make use of previous ideas from [12] and [4].

If $\gamma \in \mathrm{M}_{\mathrm{inv}}(d, \mathbb{Z})$ and $m \in \mathbb{N}$ is fixed we write

$$
\gamma \equiv \mathrm{Id}(\bmod \mathrm{m}) \Leftrightarrow \gamma-\mathrm{Id}=m A,
$$

with $A \in \mathrm{M}(d, \mathbb{Z}):=\{d \times d$ matrices with integer entries $\}$.

For a fixed $m \in \mathbb{N}$ define

$$
\Gamma^{(m)}=\{\gamma \in \Gamma: \gamma \equiv \operatorname{Id}(\bmod m)\} .
$$

We observe that $\Gamma$ acts naturally on the finite set $(\mathbb{Z} / m \mathbb{Z})^{d}$. We denote by $\gamma \mapsto \bar{\gamma}$ the corresponding homomorphism of $\Gamma$ into the semigroup $\Lambda_{m, d}$ of maps of $(\mathbb{Z} / m \mathbb{Z})^{d}$ into itself and we write

$$
\Gamma_{m}=\left\{\bar{\gamma} \in \Lambda_{m, d}: \gamma \in \Gamma\right\} .
$$

The proof depends on the following

Lemma 6.1. Assume that $\Gamma \subset \mathrm{M}_{\mathrm{inv}}(d, \mathbb{Z})$ is finitely generated and satisfies $\left(H_{1}\right)$ and $\left(H_{2}\right)$. Let $m$ be a prime number not dividing the elements of the multiplicative semigroup $\{\operatorname{det} \gamma: \gamma \in \Gamma\}$. Then $\Gamma$ acts on $(\mathbb{Z} / m \mathbb{Z})^{d}$ as a group of permutations and the semigroup $\Gamma^{(m)}=\{\gamma \in \Gamma: \gamma \equiv \operatorname{Id}(\bmod m)\}$ satisfies $\left(H_{1}\right)$ and $\left(H_{2}\right)$.

Proof. Here $\mathbb{Z} / m \mathbb{Z}$ is a finite field and for $\gamma \in \Gamma, \bar{\gamma}$ is an endomorphism of the vector space $(\mathbb{Z} / m \mathbb{Z})^{d}$. Then $\operatorname{det} \bar{\gamma}$ is the congruence class of $\operatorname{det} \gamma$ in $\mathbb{Z} / m \mathbb{Z}$. Since $m$ is a prime number not dividing $\operatorname{det} \gamma$, we conclude that $\operatorname{det} \bar{\gamma} \neq \overline{0}$, hence $\bar{\gamma} \in \operatorname{GL}(d, \mathbb{Z} / m \mathbb{Z})$. Thus $\Gamma_{m}$ is a semigroup contained in the finite group $\mathrm{GL}(d, \mathbb{Z} / m \mathbb{Z})$; it follows that $\Gamma_{m}$ is a group. We write $\Gamma_{m}=\left\{\bar{a}_{i}: a_{i} \in \Gamma, i=1, \ldots, q\right\}$, and we observe that the inverse of $\bar{a}_{i}$ is of the form $\bar{a}_{i^{\prime}}$ with $a_{i^{\prime}} \in \Gamma$ and $1 \leq i^{\prime} \leq q$. Since for every $\gamma \in \Gamma$, we have $\bar{\gamma}=\bar{a}_{i}$ for some $i$, we get $\bar{a}_{i^{\prime}} \bar{\gamma}=\mathrm{Id}, a_{i^{\prime}} \gamma \in \Gamma^{(m)}$. 
Assume condition $\left(H_{1}\right)$ is not satisfied by $\Gamma^{(m)}$; then for some subspace $W \subset V$, the orbit $\Gamma^{(m)} W$ is finite, hence so is the set $\left\{a_{i^{\prime}} \gamma W: \bar{a}_{i^{\prime}} \in \Gamma_{m}\right.$, $\left.\gamma \in \Gamma, \bar{a}_{i^{\prime}} \bar{\gamma}=\mathrm{Id}\right\}$. It follows that the set $\{\gamma W: \gamma \in \Gamma\}$ is finite, and this contradicts condition $\left(H_{1}\right)$ for $\Gamma$. Hence $\Gamma^{(m)}$ satisfies condition $\left(H_{1}\right)$.

Let $\gamma \in \Gamma$ be a proximal element of $\Gamma$. Since the group $\Gamma_{m}$ is finite, it follows that for $k=\left|\Gamma_{m}\right|$, we have $\bar{\gamma}^{k}=\mathrm{Id}$, hence $\gamma^{k} \in \Gamma^{(m)}$. Clearly, $\gamma^{k}$ is proximal.

The following lemma will also be used. Its proof is analogous to the classical case of one endomorphism of $\mathbb{T}^{d}$ (see for example [2]). In this lemma, the torus $\mathbb{T}^{d}$ is endowed with its normalized Haar measure, which is $\Gamma$ invariant.

Lemma 6.2. Assume $\Gamma \subset \mathrm{M}_{\mathrm{inv}}(d, \mathbb{Z})$ and $\Sigma \subset \mathbb{T}^{d}$ is measurable, has positive measure and satisfies $\Gamma \Sigma \subset \Sigma$. If any character $\chi \neq \mathrm{Id}$ has unbounded $\Gamma^{t}$-orbit, then $\Sigma$ has measure 1 ; in particular $\Gamma$ is ergodic on $\mathbb{T}^{d}$.

In order to prove Theorem 1.1, we can suppose $\Gamma$ to be finitely generated. In fact, Proposition 2.6 implies that $\Gamma$ contains a finitely generated semigroup $\Gamma_{1}$ which satisfies $\left(H_{1}\right)$ and $\left(H_{2}\right)$.

Since $\Sigma$ is infinite and closed, it contains limit points. We have two cases.

CASE 1: Some limit point of $\Sigma$ is rational. So, let $p / q$ be a limit point of $\Sigma$. Then the set $q \Sigma$ is $\Gamma$-invariant and has 0 as its limit point. Therefore, by considerations in Subsection 6.1 we find that $q \Sigma=\mathbb{T}^{d}$. Hence, $\Sigma$ has positive Haar measure (greater than $\left.(1 / q)^{d}\right)$. Since $\Gamma$ satisfies $\left(H_{1}\right)$ and, by Remark 2.1(ii), also $\left(H_{0}\right)$, we infer from Remark 2.1(iv) that $\Gamma^{t}$ satisfies $\left(H_{0}\right)$, hence Lemma 6.2 allows us to conclude that $\Sigma$ has measure 1. Since $\Sigma$ is closed, we have $\Sigma=\mathbb{T}^{d}$.

CASE 2: Every limit point of $\Sigma$ is irrational. Let $\Sigma^{\text {ac }}$ be the set of limit points of $\Sigma$. For $m$ fixed and prime not dividing the elements of the finitely generated semigroup $\{\operatorname{det} \gamma: \gamma \in \Gamma\}$, let $\Sigma^{(m)} \subset \Sigma^{\text {ac }}$ be a minimal $\Gamma^{(m)}$-invariant set. Since $\Sigma^{\text {ac }}$ consists of irrational points, $\Sigma^{(m)}$ is infinite, hence 0 is a limit point of the closed $\Gamma^{(m)}$-invariant subset $\Sigma^{(m)}-\Sigma^{(m)}$. From Lemma 6.1 above and considerations in Subsection 6.1 we deduce that $\Sigma^{(m)}-\Sigma^{(m)}=\mathbb{T}^{d}$. Therefore, for every $r=\left(r_{1}, \ldots, r_{d}\right) \in \mathbb{Z}^{d}$ there are $x$ and $y$ in $\Sigma^{(m)}$ such that

$$
x-y=\left(r_{1} / m, \ldots, r_{d} / m\right)=r / m .
$$

Define

$$
\Sigma_{r}^{(m)}=\left\{x \in \Sigma^{(m)}: \exists y \in \Sigma^{(m)}, x-y=r / m\right\} .
$$

Clearly, $\Sigma_{r}^{(m)}$ is closed and non-empty. Since $r / m$ is fixed by $\Gamma^{(m)}$ it follows 
that $\Sigma_{r}^{(m)}$ is $\Gamma^{(m)}$-invariant. Thus, by minimality of $\Sigma^{(m)}$ we get $\Sigma^{(m)}=$ $\Sigma_{r}^{(m)}$. Therefore, for every $m \in \mathbb{N}, x \in \Sigma^{(m)}$ and $r \in \mathbb{Z}^{d}$ we have

$$
x+r / m=y \in \Sigma^{(m)} \text {. }
$$

Hence $\Sigma^{(m)}$ is invariant under translations in $\mathbb{T}^{d}$ by $r / m, r \in \mathbb{Z}^{d}$. It follows that $\Sigma^{(m)}$ is $1 / m$-dense, hence $\Sigma^{\text {ac }}$ is $1 / m$-dense for every prime $m$ as above. We observe that the set of such primes is infinite, thus $1 / m$ can be chosen arbitrarily small. Since $\Sigma^{\mathrm{ac}}$ is closed we have $\Sigma^{\mathrm{ac}}=\mathbb{T}^{d}$, which contradicts the hypothesis. Thus, only Case 1 is possible, and hence $\Sigma=\mathbb{T}^{d}$.

\section{References}

[1] H. Abels, G. A. Margulis and G. A. Sơ̌fer, Semigroups containing proximal linear maps, Israel J. Math. 91 (1995), 1-30.

[2] M. B. Bekka and M. Mayer, Ergodic Theory and Topological Dynamics of Group Actions on Homogeneous Spaces, London Math. Soc. Lecture Note Ser. 269, Cambridge Univ. Press, 2000.

[3] Y. Benoist, Propriétés asymptotiques des groupes linéaires. II, in: Analysis on Homogeneous Spaces and Representation Theory of Lie Groups (Okayama-Kyoto, 1997), Adv. Stud. Pure Math. 26, Math. Soc. Japan, Tokyo, 2000, 33-48.

[4] D. Berend, Multi-invariant sets on tori, Trans. Amer. Math. Soc. 280 (1983), 509532.

[5] R. Bowen, Equilibrium States and the Ergodic Theory of Anosov Diffeomorphisms, Lecture Notes in Math. 470, Springer, Berlin, 1975.

[6] J.-P. Conze and Y. Guivarc'h, Limit sets of groups of linear transformations, Sankhyā Ser. A, Special issue on Ergodic Theory and Harmonic Analysis in honour of M. G. Nadkarni, 62 (2000), 367-385.

[7] —, - Densité d'orbites d'actions de groupes linéaires et propriétés d'équidistribution de marches aléatoires, in: Rigidity in Dynamics and Geometry (Cambridge, 2000), Springer, Berlin, 2002, 39-76.

[8] F. Dal'bo, Topologie du feuilletage fortement stable, Ann. Inst. Fourier (Grenoble) 50 (2000), 981-993.

[9] S. G. Dani and S. Raghavan, Orbits of Euclidean frames under discrete linear groups, Israel J. Math., 36 (1980), 300-320.

[10] B. de Saporta, Y. Guivarc'h and E. Le Page, On the multidimensional stochastic equation $Y_{n+1}=A_{n} Y_{n}+B_{n}$, C. R. Acad. Sci. Paris Sér. I 339 (2004), 499-502.

[11] P. Eberlein, Geodesic flows on negatively curved manifolds. II, Trans. Amer. Math. Soc. 178 (1973), 57-82.

[12] H. Furstenberg, Disjointness in ergodic theory, minimal sets, and a problem in Diophantine approximation, Math. Systems Theory 1 (1967), 1-49.

[13] - Boundary theory and stochastic processes on homogeneous spaces, in: Harmonic Analysis on Homogeneous Spaces, C. C. Moore (ed.), Proc. Sympos. Pure Math. 26, Amer. Math. Soc., 1972, 193-229.

[14] I. Ya. Goldsheid and Y. Guivarc'h, Zariski closure and the dimension of the Gaussian law of the product of random matrices. I, Probab. Theory Related Fields 105 (1996), 109-142. 
[15] Y. Guivarc'h, Produits de matrices aléatoires et applications aux propriétés géométriques de sous-groupes du groupe linéaire, Ergodic Theory Dynam. Systems 10 (1990), 483-512.

[16] Y. Guivarc'h et E. Le Page, Simplicité de spectres de Lyapunov et propriété d'isolation spectrale pour une famille d'opérateurs de transfert sur l'espace projectif, in: Random Walks and Geometry (Vienna, 2001), V. Kaimanovich (ed.), de Gruyter, 2004, 181-259.

[17] Y. Guivarc'h and A. Raugi, Products of random matrices: convergence theorems, in: Random Matrices and Their Applications (Brunswick, ME, 1984), Contemp. Math. 50, Amer. Math. Soc., Providence, RI, 1986, 31-54.

[18] -, - Frontière de Furstenberg, propriétés de contraction et théorèmes de convergence, Z. Wahrsch. Verw. Gebiete 69 (1985), 187-242.

[19] Y. Guivarc'h and A. N. Starkov, Orbits of linear group actions, random walks on homogeneous spaces and toral automorphisms, Ergodic Theory Dynam. Systems 24 (2004), 767-802.

[20] G. H. Hardy and J. E. Littlewood, Some problems of Diophantine approximation, Acta Math. 37 (1914), 155-191.

[21] C. T. Ionescu-Tulcea et G. Marinescu, Théorie ergodique pour une classe d'opérateurs non complètement continus, Ann. of Math. 52 (1950), 140-147.

[22] H. Kesten, Random difference equations and renewal theory for products of random matrices, Acta Math. 131 (1973), 207-248.

[23] S. P. Lalley, Renewal theorems in symbolic dynamics with application to geodesic flows, non-Euclidean tessellations and their fractal limits, ibid. 163 (1989), 1-55.

[24] G. A. Margulis, Problems and conjectures in rigidity theory, in: Mathematics: Frontiers and Perspectives, Amer. Math. Soc., Providence, RI, 2000, 161-174.

[25] R. Muchnik, Orbits of Zariski dense semigroups of $\mathrm{SL}(n, \mathbb{Z})$, Ergodic Theory Dynam. Systems, to appear.

[26] - Semigroup actions on $\mathbb{T}^{n}$, Geom. Dedicata, to appear.

[27] A. L. Onishchik and E. B. Vinberg, Lie Groups and Algebraic Groups, Springer Ser. in Soviet Math., Springer, Berlin, 1990.

[28] M. Rosenblatt, Equicontinuous Markov operators, Theory Probab. Appl. 2 (1964), 180-197.

[29] A. N. Starkov, Orbit closures of toral automorphism groups, preprint, Moscow, 1999.

\section{IRMAR}

Université de Rennes 1

Campus de Beaulieu

35042 Rennes Cedex, France

E-mail: yves.guivarch@univ-rennes1.fr
Institute of Mathematics

Wrocław University

Plac Grunwaldzki $2 / 4$

50-384 Wrocław, Poland

E-mail: urban@math.uni.wroc.pl

Received October 25, 2004

Revised version April 7, 2005 